\title{
On a Well-Conditioned Electric Field Integral Operator for Multiply Connected Geometries
}

Francesco P. Andriulli, Senior Member, IEEE, Kristof Cools, Ignace Bogaert, and Eric Michielssen, Fellow, IEEE

\begin{abstract}
All known integral equation techniques for simulating scattering and radiation from arbitrarily shaped, perfect electrically conducting objects suffer from one or more of the following shortcomings: (i) they give rise to ill-conditioned systems when the frequency is low (ii) and/or when the discretization density is high, (iii) their applicability is limited to the quasi-static regime, (iv) they require a search for global topological loops, (v) they suffer from numerical cancelations in the solution when the frequency is very low. This work presents an equation that does not suffer from any of the above drawbacks when applied to smooth and closed objects. The new formulation is obtained starting from a Helmholtz decomposition of two discretizations of the electric field integral operator obtained by using RWGs and dual bases respectively. The new decomposition does not leverage Loop and Star/Tree basis functions, but projectors that derive from them. Following the decomposition, the two discretizations are combined in a Calderon-like fashion resulting in a new overall equation that is shown to exhibit self-regularizing properties without suffering from the limitations of existing formulations. Numerical results show the usefulness of the proposed method both for closed and open structures.
\end{abstract}

Index Terms-Integral Equations, Loop-Star/Tree bases, EFIE, MFIE, Calderón Equations.

\section{INTRODUCTION}

$\mathbf{I}$ NTEGRAL equation solvers are widely used for simulating electromagnetic scattering and radiation from arbitrarily shaped, Perfect Electrically Conducting (PEC) objects. Long popular in academic circles, these solvers recently have been incorporated into several commercial electromagnetic analysis and design tools as well. Their attractiveness stems from the fact that they only require surface discretizations, operate on (comparatively) small interaction matrices that can be applied rapidly to arbitrary vectors by fast multipole and related algorithms [1], [2], [3], and yield solutions that automatically satisfy the radiation condition.

Among the many available alternatives, the surface Electric Field Integral Equation (EFIE) plays a dominant role. Although the EFIE initially was developed for simulating scattering and radiation from PEC surfaces, its underlying Electric Field Integral Operator (EFIO) also is used in integral

This work was supported in part by the Agence Nationale de la Recherche under the Grant ANR Blanc JCJC2012 FASTEEG, by the European Commission under the Grant Marie Curie GIC NEUROIMAGEEG, and by the Foundation Telecom under the Grant "Futur et Ruptures" CPCR11322.

F. P. Andriulli is with the Microwave Department of Telecom Bretagne Institut Mines-Telecom, Brest, France.

K. Cools is with the Electrical Systems and Optics Research Division, University of Nottingham, Nottingham NG7 2RD, U.K.

I. Bogaert is with the Department of Information Technology, Ghent University, Ghent 9000, Belgium

E. Michielssen is with the Department of Electrical Engineering and Computer Science, University of Michigan, Ann Arbor, MI 48109 USA. equations applicable to resistive, surface impedance, and penetrable surfaces. This explains the large effort of the scientific community, currently underway, to stably discretize and invert the EFIO, a process that is plagued by numerous problems.

When the EFIO is discretized with boundary elements with average diameter $h$, the resulting matrix has a condition number (the ratio of the matrix' largest and smallest singular values) that grows as $(k h)^{-2}$, where $k$ is the wavenumber (see [4] and references therein). When using an iterative method to solve an ill-conditioned linear system, the number of iterations tends to be large and the solution inaccurate. As a result, when $k$ approaches zero, the EFIE becomes increasingly difficult and sometimes impossible to solve. This so-called low frequency breakdown phenomenon traditionally has been remedied by using Loop-Star/Tree (quasi-Helmholtz, or Hodge) decompositions [5], [6], [7], [8], [9]. When using these decompositions with the EFIO and after appropriate matrix scaling with suitably chosen powers of $(k h)$, the low frequency breakdown is solved; that is, in the limit of $k$ going to zero, the matrix condition number is constant.

That said, these methods do not cure the undesirable scaling of the matrix condition number with $h$. Following their application, the matrix condition number scales as $h^{-1}, h^{-2}$, or $h^{-3}$ (depending on the formulation). This dense discretization breakdown phenomenon is due to the combined effect of the spectral properties of the EFIO [10], [4] and the instability of the Loop-Star/Tree bases [11].

In addition to suffering from dense discretization breakdown, Loop-Star/Tree decompositions also require the detection of global loops when the surface is a non-simply connected geometry, i.e. it contains holes and handles [5]. Existing general-purpose algorithms for finding global loops exhibit quadratic complexity. Their cost therefore scales worse than that of fast integral equation solvers, which exhibit quasilinear complexity.

Recently a new family of augmented equations that is immune to low frequency breakdown and that, remarkably, does not require the detection of global loops has been introduced [12]. Even more recently, a clever approach based on the solution of a moderately low-frequency EFIE problem, allows for a solution of the EFIE that is also immune from the lowfrequency breakdown [13]. Unfortunately, these formulations still suffer from dense discretization breakdown, since they inherit the spectral properties of the EFIO.

To protect an EFIE against both low frequency and the dense discretization breakdown, a simple rescaling of the EFIO does not suffice. Instead, a more invasive procedure aimed at modifying its spectrum is called for. This can be achieved 
by using hierarchical quasi-Helmholtz decompositions [14], [15], [16], [4] and/or Calderón techniques [17], [18], [19], [10], [20], [21], or the Epstein-Greengard method based on generalized Debye sources [22]. On a related note, the work in [17] uses a discrete Helmholtz-Hodge decomposition to construct a valid dual boundary element space for use with Calderón formulas. It hints at the fact that Helmholtz-Hodge decompositions and Calderón techniques nicely fit, and it is a premonitory fact that the scheme we present in this work succeeds.

When analyzing non-simply connected geometries, hierarchical schemes explicitly call for global loops in their current expansions and Calderón schemes require the construction of global loops at very low frequencies due to the existence of the toroidal and poloidal static null-spaces of the internal and external magnetic field operator [23]. At very low frequencies both solver families therefore require the construction of global loops. A similar problem stands for the Epstein-Greengard method.

Finally, several of the above schemes are susceptible to very low frequency cancelations in the solution vector. In fact, even if the equations are made well-conditioned, for plane wave scattering problems the physics dictates that the nonsolenoidal and solenoidal components of the current scale as $k$ and are frequency independent, respectively. If these two components are not separated during the solution process, numerical cancelations that deteriorate the accuracy of the far field computation ensue. This phenomenon has been first pointed out in [24], and further studied in [25], [26] and [27] and [28]. The reader should be aware of the meaning of "very low frequency" in this context. The above discussed low frequency breakdown, for a structure of few centimeters in size, typically kicks in when the frequency is on the order of few MHz. For the same structure, the very low frequency cancelation of the current only becomes problematic at a few Hz. This is why Calderón techniques can be successfully applied to the analysis of most scattering problems, only requiring special care when approaching statics. The very low frequency cancellation problem can be solved either by using perturbation methods [24], [25], [29] or by augmenting a Calderón scheme with Loop-Star/Tree decompositions [20], [21]. Perturbation methods are a valuable solution, but require one to switch formulations when passing from the low to the high frequency regime - their application in the latter regime would require too many terms in the perturbation series. Moreover, they inherit the spectral problems of the starting equations, that is dense discretization breakdown when applied to the augmented EFIE or the necessity of global loop extraction when applied to the MFIE or to the Calderón EFIE. The use of Loop-Star/Tree decompositions on the other hand requires the extraction of global loops and regularization of the dense discretization breakdown related to the instability of the decomposition (present in decomposed Calderón equations in [21] and [11]).

In summary, to the best of our knowledge, there exists no integral equations that is simultaneously immune to low frequency and dense discretization breakdown, and free from very low frequency cancelation and the need to detect global loops. This paper presents a new equation that does not suffer from any of these drawbacks. It introduces a new basisfree Loop-Star decomposition that derives from projections and is used to rescale the standard EFIO and remove low frequency breakdown and very low frequency current cancelation phenomena. Moreover, the rescaled EFIO is selfregularizing, and when squared in a Calderon-like fashion, is immune from dense discretization breakdown. Different from the standard Calderón EFIO, however, our new operator does not have any static null-space. In conclusion, the resulting equation simultaneously is free from low frequency and dense discretization breakdown, very low frequency cancelations, and the need to detect global loops.

This paper is organized as follows. Section II presents background material and introduces notation. Section III presents the new equation. Section IV analyzes the new equation's properties. Section V focuses on implementation details. Section VI presents numerical results that demonstrate the effectiveness of the proposed scheme. Section VII presents our conclusions and avenues for future research.

\section{BACKGROUND AND NOTATION}

Let $\Gamma$ be the surface of an orientable PEC object residing in a background medium of permittivity $\epsilon$ and permeability $\mu$ and let $\hat{\boldsymbol{n}}_{\boldsymbol{r}}$ denote $\Gamma$ 's normal vector at $\boldsymbol{r}$. Surface $\Gamma$ can be non-simply connected, i.e. it can potentially have holes and/or handles. The incident electric field $\boldsymbol{E}^{i}(\boldsymbol{r})$ impinges on $\Gamma$ and induces the surface current density $\boldsymbol{J}(\boldsymbol{r})$, which satisfies the EFIE

$$
\mathcal{T}(\boldsymbol{J})=-\hat{\boldsymbol{n}}_{\boldsymbol{r}} \times \boldsymbol{E}^{i}
$$

where $\mathcal{T}(\boldsymbol{J})=k \mathcal{T}_{s}(\boldsymbol{J})+\frac{1}{k} \mathcal{T}_{h}(\boldsymbol{J})$ with

$$
\begin{aligned}
& \mathcal{T}_{s}(\boldsymbol{J})=i \hat{\boldsymbol{n}}_{\boldsymbol{r}} \times \int_{\Gamma} \frac{e^{i k\left|\boldsymbol{r}-\boldsymbol{r}^{\prime}\right|}}{4 \pi\left|\boldsymbol{r}-\boldsymbol{r}^{\prime}\right|} \boldsymbol{J}\left(\boldsymbol{r}^{\prime}\right) \mathrm{d} \boldsymbol{r}^{\prime}, \\
& \mathcal{T}_{h}(\boldsymbol{J})=i \hat{\boldsymbol{n}}_{\boldsymbol{r}} \times \nabla \int_{\Gamma} \frac{e^{i k\left|\boldsymbol{r}-\boldsymbol{r}^{\prime}\right|}}{4 \pi\left|\boldsymbol{r}-\boldsymbol{r}^{\prime}\right|} \nabla_{s} \cdot \boldsymbol{J}\left(\boldsymbol{r}^{\prime}\right) \mathrm{d} \boldsymbol{r}^{\prime},
\end{aligned}
$$

and the wavenumber $k=2 \pi / \lambda=\omega \sqrt{\epsilon \mu}$. Note that with these definitions $\boldsymbol{J}(\boldsymbol{r})$ is the jump across $\Gamma$ of the (rotated) total magnetic field multiplied by the medium characteristic impedance $\eta=\sqrt{\mu / \epsilon}$.

To solve the EFIE by the boundary element method, $\Gamma$ is approximated by a mesh of planar triangles with average edge length $h$, and $\boldsymbol{J}(\boldsymbol{r})$ is approximated as

$$
\boldsymbol{J}(\boldsymbol{r}) \approx \sum_{n=1}^{N} I_{n} \boldsymbol{f}_{n}(\boldsymbol{r})
$$

where $\boldsymbol{f}_{n}(\boldsymbol{r}), n=1, \ldots, N$ are Rao-Wilton-Glisson (RWG) div-conforming basis functions defined on the mesh's $N$ internal edges [30]. To simplify the notation, the RWGs are defined without edge length normalization: for two adjacent triangles $c_{j}^{+}$and $c_{j}^{-}$with areas $A_{c_{j}^{+}}$and $A_{c_{j}^{-}}$and free vertices vectors $\boldsymbol{r}_{j}^{+}$and $\boldsymbol{r}_{j}^{+}$(Fig. 1), $\boldsymbol{f}_{n}$ is defined as

$$
\boldsymbol{f}_{n}(\boldsymbol{r})=\left\{\begin{array}{ll}
\frac{\boldsymbol{r}-\boldsymbol{r}_{n}^{+}}{2 A_{c_{n}^{+}}} & \text {for } \boldsymbol{r} \in c_{n}^{+} \\
\frac{\boldsymbol{r}_{n}^{-}-\boldsymbol{r}}{2 A_{c_{n}^{-}}} & \text {for } \boldsymbol{r} \in c_{n}^{-}
\end{array} .\right.
$$




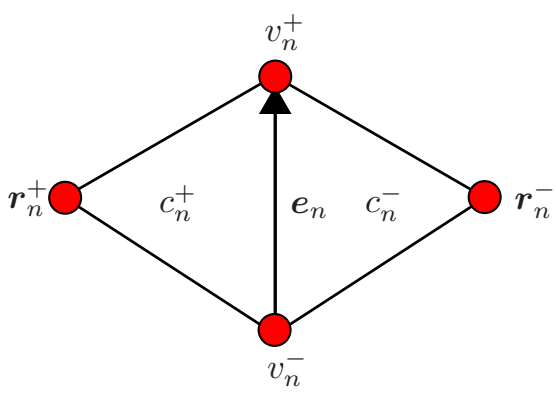

Fig. 1. Conventions used in the definition of RWG, Loop, and Star functions.

After substistuting (4) into (1), the EFIE is tested with the functions $\hat{\boldsymbol{n}}_{\boldsymbol{r}} \times \boldsymbol{f}_{i}$ yielding the $N \times N$ discretized EFIE system

$$
\overline{\overline{\mathbf{Z}}} \overline{\mathbf{I}}=\left(k \overline{\overline{\mathbf{Z}}}_{s}+\frac{1}{k} \overline{\overline{\mathbf{Z}}}_{h}\right) \overline{\mathbf{I}}=\overline{\mathbf{V}}
$$

where $\left(\overline{\overline{\mathbf{Z}}}_{s}\right)_{i, j}=\left\langle\hat{\boldsymbol{n}}_{\boldsymbol{r}} \times \boldsymbol{f}_{i}, \mathcal{T}_{s}\left(\boldsymbol{f}_{j}\right)\right\rangle, \quad\left(\overline{\overline{\mathbf{Z}}}_{h}\right)_{i, j}=$ $\left\langle\hat{\boldsymbol{n}}_{\boldsymbol{r}} \times \boldsymbol{f}_{i}, \mathcal{T}_{h}\left(\boldsymbol{f}_{j}\right)\right\rangle, \quad(\overline{\mathbf{V}})_{i}=\left\langle\boldsymbol{f}_{i}, \hat{\boldsymbol{n}}_{\boldsymbol{r}} \times \hat{\boldsymbol{n}}_{\boldsymbol{r}} \times \boldsymbol{E}^{i}\right\rangle$, and $(\overline{\mathbf{I}})_{j}=I_{j}$. Here $\langle a, b\rangle=\int_{\Gamma} a \cdot b \mathrm{~d} \Gamma$. Upon solving (6) for $\overline{\mathbf{I}}, \boldsymbol{J}$ can be approximated using (4). It should be noted that, a scale factor of $\eta$ aside, (6) is identical to the standard EFIE system of equations in [30]. The EFIO can be alternatively discretized by using the Buffa-Christiansen (BC) basis functions defined on the mesh's barycentric refinement [31]. These functions, similar to RWGs, are div-conforming and defined on the mesh's $N$ internal edges. In what follows, they are denoted by $\boldsymbol{f}_{j}^{B C}$. The BC functions are also quasi curl-conforming in the sense that the mixed gram matrix $\overline{\overline{\mathbf{G}}}_{\text {mix }}$ between BC and curl-conforming rotated RWGs

$$
\left(\overline{\overline{\mathbf{G}}}_{m i x}\right)_{i, j}=\left\langle\hat{\boldsymbol{n}}_{\boldsymbol{r}} \times \boldsymbol{f}_{i}, \boldsymbol{f}_{j}^{B C}\right\rangle .
$$

is well-conditioned. The explicit definition and further details regarding these functions can be found in [31] and [10]. The BC discretized EFIO will be denoted

$$
\overline{\overline{\mathbb{Z}}}=\left(k \overline{\overline{\mathbb{Z}}}_{s}+\frac{1}{k} \overline{\overline{\mathbb{Z}}}_{h}\right)
$$

where $\left(\overline{\mathbb{Z}}_{s}\right)_{i, j}=\left\langle\hat{\boldsymbol{n}}_{\boldsymbol{r}} \times \boldsymbol{f}_{i}^{B C}, \mathcal{T}_{s}\left(\boldsymbol{f}_{j}^{B C}\right)\right\rangle$ and $\left(\overline{\overline{\mathbb{Z}}}_{h}\right)_{i, j}=$ $\left\langle\hat{\boldsymbol{n}}_{\boldsymbol{r}} \times \boldsymbol{f}_{i}^{B C} \mathcal{T}_{h}\left(\boldsymbol{f}_{j}^{B C}\right)\right\rangle$.

To construct the new EFIE, we need to define Loop and Star transformation matrices. Since Loop and Star bases are very well-known [5], [6], [7], [8], [9], the treatment will be minimal and solely meant to introduce notation.

Loop functions are denoted by $\left\{\boldsymbol{\Lambda}_{j}, j=1, \ldots, N_{v}\right\}$ where $N_{v}$ is the number of inner vertices of the mesh. Each Loop function can be expressed as $\boldsymbol{\Lambda}_{j}=\nabla \times \hat{\boldsymbol{n}}_{\boldsymbol{r}} \lambda_{j}$, where $\lambda_{j}$ is the piecewise linear Lagrange basis function that equals one on the $j$-th inner node of the mesh and zero on all other nodes. The Loop to RWG transformation matrix, i.e. the matrix whose columns are the coefficients of Loop functions when expressing them as linear combinations of RWG functions is denoted by $\overline{\bar{\Lambda}}$. With the conventions of Fig. 1, the matrix $\overline{\bar{\Lambda}}$ can be expressed as

$$
\overline{\bar{\Lambda}}_{i, j}=\left\{\begin{aligned}
1 & \text { if node } j \text { equals } v_{i}^{+} \\
-1 & \text { if node } j \text { equals } v_{i}^{-} \\
0 & \text { otherwise }
\end{aligned}\right.
$$

If the surface $\Gamma$ is closed and if (for notational simplicity) we assume $\Gamma$ to be a single scatterer (a body with only one connected component), the set of Loop functions defined on all the vertices of the mesh is not linearly independent. The matrix $\overline{\bar{\Lambda}}$ has a one-dimensional null-space spanned by the vector $\overline{\mathbf{1}}^{\Lambda}$ of length $N_{v}$ containing all ones, i.e.

$$
\overline{\bar{\Lambda}} \overline{\mathbf{1}}^{\Lambda}=\overline{\mathbf{0}} \text {. }
$$

The Star functions are denoted by $\left\{\boldsymbol{\Sigma}_{j}, j=1, \ldots, N_{c}\right\}$ where $N_{c}$ is the number of cells of the mesh. The Star to RWG transformation matrix, i.e. the matrix whose columns are the coefficients of Star functions when expressing them as linear combinations of RWG functions, is denoted by $\overline{\bar{\Sigma}}$. With the conventions of Fig. 1, the matrix $\overline{\bar{\Sigma}}$ can be expressed as

$$
\overline{\bar{\Sigma}}_{i, j}=\left\{\begin{aligned}
1 & \text { if the cell } j \text { equals } c_{i}^{+} \\
-1 & \text { if the cell } j \text { equals } c_{i}^{-} \\
0 & \text { otherwise }
\end{aligned}\right.
$$

The set of Star functions is not linearly independent. The matrix $\overline{\bar{\Sigma}}$ has a one-dimensional null-space spanned by the vector $\overline{\mathbf{1}}^{\Sigma}$ of length $N_{c}$ containing all ones, i.e.

$$
\overline{\bar{\Sigma}} \overline{\mathbf{1}}^{\Sigma}=\overline{\mathbf{0}} \text {. }
$$

From their definitions it follows immediately that the Loop and Star transformation matrices are mutually orthogonal [6], i.e.

$$
\overline{\bar{\Sigma}}^{T} \overline{\overline{\mathbf{\Lambda}}}=\overline{\overline{\mathbf{0}}}
$$

It should be noted that the particularly simple form of the Loop and Star to RWG transformation matrices in (9) and in (11) is a consequence of (5), where the RWGs are defined without edge length normalization.

When $\Gamma$ is simply connected, the Loop and Star functions span the entire RWG space, i.e. given an arbitrary RWG coefficient vector $\overline{\mathbf{I}}$ there exist two (in general non unique) vectors $\overline{\mathrm{l}}$ and $\overline{\mathrm{s}}$ such that

$$
\overline{\mathbf{I}}=\overline{\bar{\Lambda}} \overline{\mathbf{l}}+\overline{\overline{\mathbf{\Sigma}}} \overline{\mathbf{s}} .
$$

When $\Gamma$ is not simply connected, the above expression should be modified in

$$
\overline{\mathbf{I}}=\overline{\overline{\mathbf{\Lambda}}} \overline{\mathbf{l}}+\overline{\overline{\mathbf{\Sigma}}} \overline{\mathbf{s}}+\overline{\overline{\mathbf{H}}} \overline{\mathbf{h}}
$$

where $\overline{\overline{\mathbf{H}}}$ is the so-called global loop to RWG transformation matrix. Global loops are the discretized counterparts of the harmonic space of the Helmholtz decomposition. It is impossible to provide a complete description of this family of functions and the interested reader is referred to [5] and [23]. The column dimension of $\overline{\overline{\mathbf{H}}}$ is $2 N_{\text {handles }}+N_{\text {holes }}$, where $N_{\text {handles }}$ and $N_{\text {holes }}$ are the number of handles and holes of $\Gamma$, respectively. The orthogonality properties

$$
\overline{\overline{\boldsymbol{\Sigma}}}^{T} \overline{\overline{\mathbf{H}}}=\overline{\overline{\mathbf{0}}} \text { and } \overline{\overline{\mathbf{\Lambda}}}^{T} \overline{\overline{\mathbf{H}}}=\overline{\overline{\mathbf{0}}} \text {. }
$$

hold. It should also be noted that both $\overline{\bar{\Lambda}} \overline{\mathbf{l}}$ and $\overline{\overline{\mathbf{H}}} \overline{\mathbf{h}}$ are RWG coefficients of solenoidal functions. Given the decomposition in (15), a dual decomposition exists for the BC functions ([31], [32], [20], [21]): given an arbitrary BC coefficient vector $\overline{\mathbb{I}}$, the following decomposition holds

$$
\overline{\mathbb{I}}=\overline{\overline{\mathbf{\Lambda}}} \overline{\mathbb{I}}+\overline{\bar{\Sigma}} \bar{\Phi}+\overline{\overline{\mathbf{H}}} \overline{\mathfrak{h}}
$$


where the two vectors $\overline{\mathbb{1}}$ and $\bar{\Phi}$ are in general not unique. It should be noted that both $\overline{\bar{\Sigma}} \bar{\Phi}$ and $\overline{\overline{\mathbf{H}}} \overline{\mathrm{h}}$ are BC coefficients of solenoidal functions.

\section{THE NEW EQUATION}

We next describe a new EFIE that, contrary to currently available ones, is concurrently immune to the low frequency breakdown and the dense discretization breakdown, as well as very low frequency solution cancelation. Moreover, the new equation will not require an identification search of global loops, that is the matrix $\overline{\mathbf{H}}$ defined above. The new formulation is obtained in two steps: (i) first, a quasi-Helmholtz decomposition is applied to the EFIO to cure it from low frequency breakdown. For this purpose, we introduce a new, basis-free decomposition, since a standard quasi-Helmholtz decomposition introduces a basis-related dense discretization breakdown (see [11]) and would require the explicit determination of $\overline{\overline{\mathbf{H}}}$ (the global loops). (ii) The new decomposed equation will be "squared" in a suitable, Calderón like fashion to cure it from dense discretization breakdown.

Space limitations do not allow for a thorough discussion of the low frequency breakdown of the EFIO and the treatment here will be minimal; further details can be found, for example, in [7]. The low frequency breakdown is due to the different scaling of the terms involving $\overline{\mathbf{Z}}_{s}$ and $\overline{\overline{\mathbf{Z}}}_{h}$ with $k$ [7]. Since both $\overline{\bar{\Lambda}} \bar{l}$ and $\overline{\mathbf{H}} \overline{\mathbf{h}}$ are RWG coefficients of solenoidal functions, it is easily verified that

$$
\overline{\overline{\mathbf{Z}}}_{h} \overline{\overline{\mathbf{\Lambda}}}=\overline{\overline{\mathbf{0}}}, \quad \overline{\overline{\mathbf{Z}}}_{h} \overline{\overline{\mathbf{H}}}=\overline{\overline{\mathbf{0}}}, \quad \overline{\overline{\mathbf{\Lambda}}}^{T} \overline{\overline{\mathbf{Z}}}_{h}=\overline{\overline{\mathbf{0}}}, \quad \overline{\overline{\mathbf{H}}}^{T} \overline{\overline{\mathbf{Z}}}_{h}=\overline{\overline{\mathbf{0}}}
$$

Then the Loop-Star decomposition solves the low frequency breakdown since, by defining $\overline{\overline{\mathbf{Z}}}^{\Lambda H \Sigma}=$ $[\overline{\bar{\Lambda}}, \overline{\overline{\mathbf{H}}}, \overline{\bar{\Sigma}}]^{T} \overline{\overline{\mathbf{Z}}}[\overline{\bar{\Lambda}}, \overline{\overline{\mathbf{H}}}, \overline{\bar{\Sigma}}]$, we have

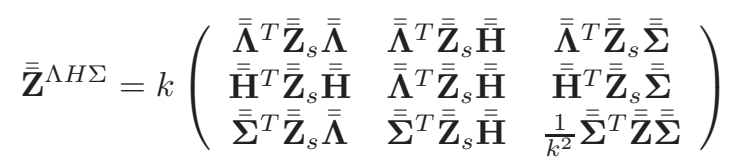

so that a simple block normalization matrix $\overline{\overline{\mathbf{D}}}=$ $\operatorname{diag}\{\sqrt{k}, \sqrt{k}, 1 / \sqrt{k}\}$ eliminates the matrix ill-scaling

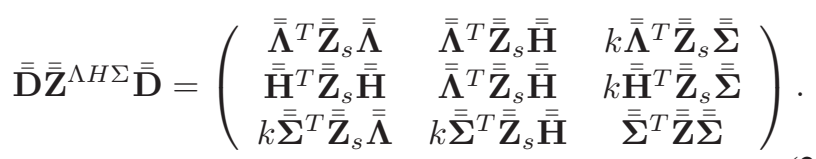

Three things should be retained by an analysis of equation (20). (i) Equation (20) still suffers from an $h$ dependent ill-conditioning due to the operator spectra of $\overline{\bar{\Lambda}}^{T} \overline{\mathbf{Z}}_{s} \overline{\bar{\Lambda}}$ and $\overline{\bar{\Sigma}}^{T} \overline{\overline{\mathbf{Z}}} \overline{\overline{\mathbf{\Sigma}}}$ and due to the instability of the Loop-Star basis, i.e. the fact that the following condition number bound

$$
\operatorname{cond}\left([\overline{\overline{\mathbf{\Lambda}}}, \overline{\overline{\mathbf{H}}}, \overline{\bar{\Sigma}}]^{T}[\overline{\overline{\mathbf{\Lambda}}}, \overline{\overline{\mathbf{H}}}, \overline{\bar{\Sigma}}]\right)>\frac{C}{h^{2}}
$$

holds [11], where $C$ is an $h$ independent constant. (ii) The combined action of $[\overline{\bar{\Lambda}}, \overline{\overline{\mathbf{H}}}, \overline{\bar{\Sigma}}] \overline{\bar{D}}$ takes each loop, global loop, and star component of the initial RWG current vector and scales them by the appropriate factor. (iii) The scaling factor for loops and global loops is the same. As a side note: the $h$ dependent ill-conditioning would have been present in (20) even if the blocks would have been scaled with $k h$ instead of $k$. Observations (ii) and (iii) suggest a different strategy to decompose the equation. Note that since $\overline{\bar{\Sigma}}^{T} \overline{\bar{\Lambda}}=\overline{\overline{\mathbf{0}}}$ and $\overline{\bar{\Sigma}}^{T} \overline{\mathbf{H}}=\overline{\overline{\mathbf{0}}}$ then for an arbitrary RWG vector $\overline{\mathbf{I}}$

$$
\overline{\overline{\boldsymbol{\Sigma}}}^{T} \overline{\mathbf{I}}=\overline{\overline{\boldsymbol{\Sigma}}}^{T} \overline{\overline{\mathbf{\Lambda}}} \overline{\mathbf{l}}+\overline{\overline{\boldsymbol{\Sigma}}}^{T} \overline{\overline{\boldsymbol{\Sigma}}} \overline{\mathbf{s}}+\overline{\overline{\boldsymbol{\Sigma}}}^{T} \overline{\overline{\mathbf{H}}} \overline{\mathbf{h}}=\overline{\overline{\boldsymbol{\Sigma}}}^{T} \overline{\overline{\mathbf{\Sigma}}} \overline{\mathbf{s}}
$$

so that if we assume $\Gamma$ to be a single scatterer we can use the degree of freedom we have in choosing $\overline{\mathbf{s}}$ to set $\overline{\mathbf{s}}^{T} \overline{\mathbf{1}}^{\Sigma}=0$, we get

$$
\overline{\mathbf{s}}=\left(\overline{\overline{\boldsymbol{\Sigma}}}^{T} \overline{\overline{\mathbf{\Sigma}}}\right)^{+} \overline{\overline{\boldsymbol{\Sigma}}}^{T} \overline{\mathbf{I}}
$$

where $\left(\overline{\overline{\boldsymbol{\Sigma}}}^{T} \overline{\overline{\boldsymbol{\Sigma}}}\right)^{+}$denotes the pseudoinverse of $\left(\overline{\overline{\boldsymbol{\Sigma}}}^{T} \overline{\overline{\boldsymbol{\Sigma}}}\right)$. Finally, the star component of $\overline{\mathbf{I}}$ is obtained by using the projection $\overline{\overline{\mathbf{P}}}^{\Sigma} \overline{\mathbf{I}}$, where the projector $\overline{\overline{\mathbf{P}}}^{\Sigma}$ is defined as

$$
\overline{\overline{\mathbf{P}}}^{\Sigma}=\overline{\overline{\boldsymbol{\Sigma}}}\left(\overline{\overline{\boldsymbol{\Sigma}}}^{T} \overline{\overline{\boldsymbol{\Sigma}}}\right)^{+} \overline{\overline{\boldsymbol{\Sigma}}}^{T}
$$

The loops and global loops components of $\overline{\mathbf{I}}$ can be obtained by the complementary projector

$$
\overline{\overline{\mathbf{P}}}^{\Lambda H}=\overline{\overline{\mathbf{I}}}-\overline{\overline{\boldsymbol{\Sigma}}}\left(\overline{\overline{\boldsymbol{\Sigma}}}{ }^{T} \overline{\overline{\boldsymbol{\Sigma}}}\right)^{+} \overline{\overline{\boldsymbol{\Sigma}}}^{T}
$$

Note that it is not necessary to discriminate further between loops and global loops, since the associated scaling factor will be the same (observation (iii) above); this renders unnecessary the explicit and costly recovery of global loops. Finally, define the decomposition operator

$$
\overline{\overline{\mathbf{M}}}=\overline{\overline{\mathbf{P}}}^{\Lambda H} \frac{1}{\sqrt{k}}+i \overline{\overline{\mathbf{P}}}^{\Sigma} \sqrt{k},
$$

and note that $\overline{\overline{\mathbf{M}}}^{T}=\overline{\overline{\mathbf{M}}}$. The role of the imaginary constant $i$ in the definition (26) will become clear in Section IV. Using the decomposition operator, the EFIO takes the form

$$
\begin{aligned}
\overline{\mathbf{M}}^{T} \overline{\overline{\mathbf{Z}}} \overline{\overline{\mathbf{M}}} & =\left(\overline{\overline{\mathbf{P}}}^{\Lambda H} \overline{\overline{\mathbf{Z}}}_{s} \overline{\overline{\mathbf{P}}}^{\Lambda H}-\overline{\overline{\mathbf{P}}}^{\Sigma} \overline{\overline{\mathbf{Z}}}_{h} \overline{\overline{\mathbf{P}}}^{\Sigma}\right) \\
& +\left(\overline{\overline{\mathbf{P}}}^{\Lambda H} \overline{\overline{\mathbf{Z}}}_{s} \overline{\overline{\mathbf{P}}}^{\Sigma}+\overline{\overline{\mathbf{P}}}^{\Sigma} \overline{\overline{\mathbf{Z}}}_{s} \overline{\overline{\mathbf{P}}}^{\Lambda H}\right) i k \\
& -\left(\overline{\overline{\mathbf{P}}}^{\Sigma} \overline{\overline{\mathbf{Z}}}_{s} \overline{\overline{\mathbf{P}}}^{\Sigma}\right) k^{2} \\
& =\left(\overline{\overline{\mathbf{P}}}^{\Lambda H} \overline{\overline{\mathbf{Z}}}_{s} \overline{\overline{\mathbf{P}}}^{\Lambda H}-\overline{\overline{\mathbf{Z}}}_{h}\right)+O(k)
\end{aligned}
$$

which is clearly immune from low frequency breakdown. In (27) we have used $\overline{\overline{\mathbf{P}}}^{\Sigma} \overline{\overline{\mathbf{Z}}}_{h} \overline{\overline{\mathbf{P}}}^{\Sigma}=\overline{\overline{\mathbf{Z}}}_{h}$ which easily follows from $\overline{\overline{\mathbf{P}}}^{\Sigma}+\overline{\overline{\mathbf{P}}}^{\Lambda H}=\overline{\overline{\mathbf{I}}}$ and $\overline{\overline{\mathbf{Z}}}_{h} \overline{\overline{\mathbf{P}}}^{\Lambda H}=\overline{\overline{\mathbf{P}}}^{\Lambda H} \overline{\overline{\mathbf{Z}}}_{h}=\overline{\overline{\mathbf{0}}}$. Note that, contrary to decomposition (20), (27) does not require the identification of global loops and, in addition, is a stable decomposition since $\overline{\overline{\mathbf{P}}}^{\Lambda H}$ and $\overline{\overline{\mathbf{P}}}^{\Sigma}$ are projectors and thus cond $\left(\overline{\mathbf{M}}^{T} \overline{\overline{\mathbf{M}}}\right)$ is independent of $h$, which compares very favorably with (21).

The treatment for $\mathrm{BC}$ discretized matrix $\overline{\overline{\mathbb{Z}}}$ is the same provided that the role of the loop and star matrices is exchanged. In fact it should be recalled that in (17) $\overline{\bar{\Sigma}} \bar{\Phi}$ and $\overline{\overline{\mathbf{H}}} \overline{\mathrm{h}}$ are BC coefficients of solenoidal functions. Thus we will define the dual projectors

$$
\begin{gathered}
\overline{\overline{\mathbb{P}}}^{\Lambda}=\overline{\overline{\mathbf{\Lambda}}}\left(\overline{\overline{\boldsymbol{\Lambda}}^{T}} \overline{\overline{\mathbf{\Lambda}}}\right)^{+} \overline{\overline{\boldsymbol{\Lambda}}}^{T} \\
\overline{\overline{\mathbb{P}}}^{\Sigma H}=\overline{\overline{\mathbf{I}}}-\overline{\overline{\mathbf{\Lambda}}}\left(\overline{\overline{\mathbf{\Lambda}}}{ }^{T} \overline{\overline{\mathbf{\Lambda}}}\right)^{+} \overline{\overline{\boldsymbol{\Lambda}}}^{T}
\end{gathered}
$$

and the associated decomposition operator

$$
\overline{\overline{\mathrm{M}}}=\overline{\overline{\mathbb{P}}}^{\Sigma H} \frac{1}{\sqrt{k}}+i \overline{\overline{\mathbb{P}}}^{\Lambda} \sqrt{k}
$$


so that the decomposed $\mathrm{BC}$-discretized EFIO reads

$$
\begin{aligned}
& \overline{\overline{\mathbf{M}}}^{T} \overline{\overline{\mathbb{Z}}} \overline{\overline{\mathbf{M}}}=\left(\overline{\overline{\mathbb{P}}}^{\Sigma H} \overline{\overline{\mathbb{Z}}}_{s} \overline{\overline{\mathbb{P}}}^{\Sigma H}-\overline{\overline{\mathbb{P}}}^{\Lambda} \overline{\overline{\mathbb{Z}}}_{h} \overline{\overline{\mathbb{P}}}^{\Lambda}\right) \\
& +\left(\overline{\overline{\mathbb{P}}}^{\Sigma H} \overline{\overline{\mathbb{Z}}}_{s} \overline{\overline{\mathbb{P}}}^{\Lambda}+\overline{\overline{\mathbb{P}}}^{\Lambda} \overline{\overline{\mathbb{Z}}}_{s} \overline{\overline{\mathbb{P}}}^{\Sigma H}\right) i k \\
& -\left(\overline{\overline{\mathbb{P}}}^{\Lambda} \overline{\overline{\mathbb{Z}}}_{s} \overline{\overline{\mathbb{P}}}^{\Lambda}\right) k^{2} \\
& =\left(\overline{\overline{\mathbb{P}}}^{\Sigma H} \overline{\overline{\mathbb{Z}}}_{s} \overline{\overline{\mathbb{P}}}^{\Sigma H}-\overline{\overline{\mathbb{Z}}}_{h}\right)+O(k)
\end{aligned}
$$

which is also immune from low frequency breakdown. The practical computation of $\left(\overline{\bar{\Sigma}}^{T} \overline{\bar{\Sigma}}\right)^{+}$and $\left(\overline{\bar{\Lambda}}^{T} \overline{\bar{\Lambda}}\right)^{+}$will be detailed in Section V. Moreover, note that if $\Gamma$ has more than one connected components, i.e. when $\Gamma$ comprises multiple scatterers, the treatment in this section goes through with only minor modifications. Specifically, the dimension of the nullspace of $\overline{\bar{\Sigma}}^{T} \overline{\bar{\Sigma}}$ will equal the number of scatterers, while the dimension of the null-space of $\overline{\bar{\Lambda}}^{T} \overline{\bar{\Lambda}}$ will equal the number of closed scatterers. Definitions (24)-(31) remain intact.

The reader notices that $\overline{\bar{\Lambda}}$ and $\overline{\bar{\Sigma}}$ are the edge-vertex and edge-cell incidence matrices of the mesh, respectively. In graph theoretical terms they stand for the topological gradients on the standard and dual meshes. The matrices $\overline{\bar{\Sigma}}^{T}$ and $\overline{\bar{\Lambda}}^{T}$ similarly represent the topological divergences on these meshes. Not surprisingly, $\overline{\bar{\Lambda}}^{T} \overline{\bar{\Lambda}}$ and $\bar{\Sigma}^{T} \bar{\Sigma}$ are the graph Laplacians of the standard and dual meshes, and the projectors $\overline{\bar{P}}^{\Lambda}=\overline{\bar{\Lambda}}\left(\overline{\bar{\Lambda}}^{T} \overline{\bar{\Lambda}}\right)^{+} \overline{\overline{\boldsymbol{\Lambda}}}^{T}$ and $\overline{\overline{\mathbf{P}}}^{\Sigma}=\overline{\bar{\Sigma}}\left(\overline{\bar{\Sigma}}^{T} \overline{\bar{\Sigma}}\right)^{+} \overline{\bar{\Sigma}}^{T}$ are the graphtheoretical counterparts of the smoothing projector $\nabla_{s} \Delta_{s}^{-1} \nabla_{s}$. in the irrotational space [33].

The EFIO, after solving the low frequency breakdown with a Loop-Star decomposition, has a condition number that still grows as an inverse power of $h$, this is the dense discretization breakdown problem (refer to [10], [4] for further details). For the standard EFIO both the low frequency and the dense discretization breakdown are solved by Calderón preconditioning: the EFIO discretized matrix $\overline{\bar{Z}}$ is replaced by the Calderón preconditioned matrix $\overline{\overline{\mathbb{Z}}} \overline{\mathbf{G}}_{m i x}^{-1} \overline{\overline{\mathbf{Z}}}$ and the Calderón preconditioned EFIE

$$
\overline{\overline{\mathbb{Z}}} \overline{\mathbf{G}}_{m i x}^{-1} \overline{\overline{\mathbf{Z}}} \overline{\mathbf{I}}=\overline{\overline{\mathbb{Z}}} \overline{\overline{\mathbf{G}}}_{m i x}^{-1} \overline{\mathbf{V}}
$$

is solved instead of solving the EFIE system in (6). The matrix $\overline{\bar{Z}} \overline{\mathbf{G}}_{\text {mix }}^{-1} \overline{\overline{\mathbf{Z}}}$ is provably immune from both low frequency and dense discretization breakdown, however it has a null-space in statics [23] and it can suffer from current cancelation at very low frequencies. The new equation proposed in this work is obtained by replacing the RWG- and BC-discretized EFIO operators $\overline{\bar{Z}}$ and $\overline{\bar{Z}}$ with the respective decomposed ones in (27) and in (31). Our new equation reads

$$
\left(\overline{\overline{\mathbf{M}}}^{T} \overline{\overline{\mathbb{Z}}} \overline{\overline{\mathbf{M}}}\right) \overline{\overline{\mathbf{G}}}_{m i x}^{-1}\left(\overline{\overline{\mathbf{M}}}^{T} \overline{\overline{\mathbf{Z}}} \overline{\overline{\mathbf{M}}}\right) \overline{\mathbf{Y}}=\left(\overline{\overline{\mathbf{M}}}^{T} \overline{\overline{\mathbb{Z}}} \overline{\overline{\mathbf{M}}}\right) \overline{\overline{\mathbf{G}}}_{m i x}^{-1} \overline{\overline{\mathbf{M}}}^{T} \overline{\mathbf{V}}
$$

with $\overline{\mathbf{I}}=(\overline{\mathbf{M}}) \overline{\mathbf{Y}}$.

\section{Properties of the New Equation}

Let's first prove that the operator of the new equation has the same null-space of the EFIO and thus, in particular, it does not have the global loops spanned static null-space of the Calderón EFIE. It is sufficient to prove the statement in statics, in fact $\overline{\overline{\mathbf{G}}}_{m i x}^{-1}$ is non-singular and, away from statics, also the matrices
$\overline{\bar{M}}$ and $\overline{\bar{M}}$ are well-defined and non-singular. Let's study the static limit of (27), i.e. let's prove that the operator

$$
\left(\overline{\overline{\mathbf{P}}}^{\Lambda H} \overline{\overline{\mathbf{Z}}}_{s} \overline{\overline{\mathbf{P}}}^{\Lambda H}-\overline{\overline{\mathbf{P}}}^{\Sigma} \overline{\overline{\mathbf{Z}}}_{h} \overline{\overline{\mathbf{P}}}^{\Sigma}\right)
$$

does not have a null-space. Since $\overline{\overline{\mathbf{P}}}{ }^{\Lambda H} \overline{\overline{\mathbf{P}}}^{\Sigma}=\overline{\overline{\mathbf{0}}}$ it is sufficient to prove that $\overline{\mathbf{P}}^{\Lambda H} \overline{\mathbf{Z}}_{s} \overline{\mathbf{P}}^{\Lambda H}$ is non-singular on the range of $[\overline{\bar{\Lambda}}, \overline{\overline{\mathbf{H}}}]$ and that $\overline{\overline{\mathbf{P}}}^{\Sigma} \overline{\overline{\mathbf{Z}}}_{h} \overline{\overline{\mathbf{P}}}^{\Sigma}$ is non singular on the range of $\overline{\overline{\mathbf{\Sigma}}}$. Since on these spaces both $\overline{\overline{\mathbf{Z}}}_{s}$ and $\overline{\overline{\mathbf{Z}}}_{h}$ in statics are symmetric positive definite matrices [34], then we get that $\forall \overline{\mathbf{v}}$ in the range of $[\overline{\bar{\Lambda}}, \overline{\overline{\mathbf{H}}}]$

$$
\begin{aligned}
\overline{\mathbf{v}}^{T} \overline{\overline{\mathbf{P}}}^{\Lambda H} \overline{\overline{\mathbf{Z}}}_{s} \overline{\overline{\mathbf{P}}}^{\Lambda H} \overline{\mathbf{v}}=0 \Rightarrow \overline{\overline{\mathbf{P}}^{\Lambda H} \overline{\mathbf{v}}=\overline{\mathbf{0}}} \\
\Rightarrow \overline{\mathbf{v}} \text { is in the range of } \overline{\overline{\mathbf{\Sigma}}} \Rightarrow \overline{\mathbf{v}}=\overline{\mathbf{0}}
\end{aligned}
$$

dually, $\forall \overline{\mathbf{q}}$ in the range of $\overline{\bar{\Sigma}}$

$$
\begin{aligned}
\overline{\mathbf{q}}^{T} \overline{\overline{\mathbf{P}}}^{\Sigma} \overline{\overline{\mathbf{Z}}}_{s} \overline{\overline{\mathbf{P}}}^{\Sigma} \overline{\mathbf{q}}=0 \Rightarrow \overline{\overline{\mathbf{P}}}^{\Sigma} \overline{\mathbf{v}}=\overline{\mathbf{0}} \\
\quad \Rightarrow \overline{\mathbf{q}} \text { is in the range of }[\overline{\overline{\mathbf{\Lambda}}}, \overline{\overline{\mathbf{H}}}] \Rightarrow \overline{\mathbf{q}}=\overline{\mathbf{0}}
\end{aligned}
$$

from this we deduce that (27) does not have a static nullspace. The same statement is proved for (31) by using the same approach. Finally we deduce that the newly proposed equation (33) has no static null-space since it is the product of three non-singular matrices.

Let's now study the conditioning behavior of (33) as a function of frequency and discretization. Since both (27) and (31) are immune from the low frequency breakdown, so will be (33), since $\overline{\mathbf{G}}_{m i x}^{-1}$ is a frequency independent matrix.

To show that the equation is also immune from the dense discretization breakdown. We will prove this in the static limit. After substituting (27) and (31) in (33) and using the relationship $\overline{\bar{Z}}_{h} \overline{\overline{\mathbf{G}}}_{m i x}^{-1} \overline{\overline{\mathbf{Z}}}_{h}=\overline{\overline{\mathbf{0}}}$, the operator of the new equation can be written as

$$
\begin{aligned}
& \left(\overline{\bar{M}}^{T} \overline{\overline{\mathbb{Z}}} \overline{\overline{\mathbf{M}}}\right) \overline{\overline{\mathbf{G}}}_{m i x}^{-1}\left(\overline{\mathbf{M}}^{T} \overline{\overline{\mathbf{Z}}} \overline{\mathbf{M}}\right)= \\
& =-\left(\overline{\overline{\mathbb{P}}}^{\Sigma H} \overline{\overline{\mathbb{Z}}}_{s} \overline{\overline{\mathbf{P}}}^{\Sigma H}\right) \overline{\overline{\mathbf{G}}}_{m i x}^{-1} \overline{\overline{\mathbf{Z}}}_{h}-\overline{\overline{\mathbb{Z}}}_{h} \overline{\overline{\mathbf{G}}}_{m i x}^{-1}\left(\overline{\overline{\mathbf{P}}}^{\Lambda H} \overline{\overline{\mathbf{Z}}}_{s} \overline{\overline{\mathbf{P}}}^{\Lambda H}\right) \\
& \quad+\left(\overline{\overline{\mathbb{P}}}^{\Sigma H} \overline{\overline{\mathbb{Z}}}_{s} \overline{\overline{\mathbb{P}}}^{\Sigma H}\right) \overline{\overline{\mathbf{G}}}_{m i x}^{-1}\left(\overline{\overline{\mathbf{P}}}^{\Lambda H} \overline{\overline{\mathbf{Z}}}_{s} \overline{\overline{\mathbf{P}}}^{\Lambda H}\right)+O(k)
\end{aligned}
$$

where the property $\overline{\bar{Z}}_{h} \overline{\overline{\mathbf{G}}}_{m i x}^{-1} \overline{\overline{\mathbf{Z}}}_{h}=\overline{\overline{\mathbf{0}}}$ has been used (see, for example, [32] and [35]). At the same time, since $\overline{\mathbf{P}}^{\Sigma}+\overline{\overline{\mathbf{P}}}^{\Lambda H}=$ $\overline{\bar{P}}^{\Lambda}+\overline{\bar{P}}^{\Sigma H}=\overline{\overline{\mathbf{I}}}$ and since, from the properties of the mixed Gram matrix we have $\overline{\bar{P}}^{\Lambda} \overline{\overline{\mathbf{G}}}_{m i x}^{-1} \overline{\overline{\mathbf{Z}}}_{h}=\left(\overline{\overline{\mathbb{P}}}^{\Lambda} \overline{\overline{\mathbf{G}}}_{m i x}^{-1} \overline{\overline{\mathbf{P}}}^{\Sigma}\right) \overline{\overline{\mathbf{Z}}}_{h}=\overline{\overline{\mathbf{0}}}$ and $\overline{\bar{Z}}_{h} \overline{\overline{\mathbf{G}}}_{m i x}^{-1} \overline{\overline{\mathbf{P}}}^{\Sigma}=\overline{\overline{\mathbb{Z}}}_{h}\left(\overline{\overline{\mathbb{P}}}^{\Lambda} \overline{\overline{\mathbf{G}}}_{m i x}^{-1} \overline{\overline{\mathbf{P}}}^{\Sigma}\right)=\overline{\overline{\mathbf{0}}}$, then the standard Calderón EFIE operator $\overline{\overline{\mathbb{Z}}} \overline{\overline{\mathbf{G}}}_{m i x}^{-1} \overline{\overline{\mathbf{Z}}}$ can be written as

$$
\overline{\overline{\mathbb{Z}}} \overline{\overline{\mathbf{G}}}_{m i x}^{-1} \overline{\overline{\mathbf{Z}}}=\overline{\overline{\mathbb{Z}}}_{h} \overline{\overline{\mathbf{G}}}_{m i x}^{-1} \overline{\overline{\mathbf{P}}}^{\Lambda H} \overline{\overline{\mathbf{Z}}}_{s}+\overline{\overline{\mathbb{Z}}}_{s} \overline{\overline{\mathbb{P}}}^{\Sigma H} \overline{\overline{\mathbf{G}}}_{m i x}^{-1} \overline{\overline{\mathbf{Z}}}_{h}+O\left(k^{2}\right)
$$

In (37), the third term can be neglected since it is a discretization of the compact operator $\mathcal{T}_{s}^{2}$. Given this it is clear that (37) maps the range of $\overline{\overline{\mathbf{P}}}^{\Lambda H}$ into the range of $\overline{\bar{P}}^{\Lambda}$ and the range of $\overline{\overline{\mathbf{P}}}^{\Sigma}$ into the range of $\overline{\overline{\mathbb{P}}}^{\Sigma H}$ and these sets are mutually orthogonal. When these sets are used to decompose both (37) and (38) the two equations will have the same diagonal blocks and the former will be block diagonal. Thus the singular values of the new equation (33) will be 
(asymptotically) bounded by the maximum and minimum singular values of the standard calderon equation. Thus the operator in (33) will have the same conditioning behavior as the standard Calderón EFIO. The reader should notice that although the term $\left(\overline{\bar{P}}^{\Sigma H} \overline{\bar{Z}}_{s} \mathbb{\overline { P }}^{\Sigma H}\right) \overline{\overline{\mathbf{G}}}_{\text {mix }}^{-1}\left(\overline{\overline{\mathbf{P}}}^{\Lambda H} \overline{\overline{\mathbf{Z}}}_{s} \overline{\overline{\mathbf{P}}}^{\Lambda H}\right)$ does not influence the $h$-dependent conditioning of (37), it is instead important for determining its null-space. In fact, it could be shown that the presence of this operator (which is absent in the static Calderón EFIO) is responsible for the absence of the static null-space of (33) (this absence has been proved earlier in this section by using another approach).

To complete the analysis of the properties of (33) we will study the frequency behavior of the solution for different types of excitation in order to show that the solution of (33) does not suffer from very low frequency cancelations. If we assume that, as a function of $k$, the solenoidal part of the physical current $\overline{\mathbf{I}}$ scales as $\left(R_{s}, I_{s}\right)$ (real part and imaginary part) and if we assume that the non-solenoidal part scales as $\left(R_{n s}, I_{n s}\right)$, then the scattered far-field due to the solenoidal part will scale as $\left(R_{s}^{F}, I_{s}^{F}\right)=\left(k I_{s}, k R_{s}\right)$ while the scattered field due to the non-solenoidal part will scale as $\left(R_{n s}^{F}, I_{n s}^{F}\right)=\left(R_{n s}, I_{n s}\right)$ [24]. Then from the relationship $\overline{\mathbf{Y}}=\overline{\mathbf{M}}^{-1} \overline{\mathbf{I}}$ and the definition of $\overline{\mathbf{M}}$ it follows that the solenoidal part of the solution $\overline{\mathbf{Y}}$ of (33) will scale as $\left(R_{s}^{Y}, I_{s}^{Y}\right)=\left(\sqrt{k} R_{s}, \sqrt{k} I_{s}\right)$ while the nonsolenoidal part will scale as $\left(R_{n s}^{Y}, I_{n s}^{Y}\right)=\left(\frac{1}{\sqrt{k}} I_{n s}, \frac{1}{\sqrt{k}} R_{n s}\right)$. We will consider the cases of plane wave excitation, inductive voltage gap excitation (the geometry is such that at least one global loop runs through the excitation port) and capacitive voltage gap excitation (the geometry is such that no global loop runs through the excitation port). For these sources we will use the scalings obtained in [26]. The current scalings as a function of $k$ are summarized in Table I. The rightmost column of the table identifies the current components that need to be recovered precisely in order to obtain a correct result for the current (and, where applicable, for the input impedance). The far-field scalings as a function of $k$ are summarized in Table II. The rightmost column of the table identifies the current components that need to be recovered precisely in order to obtain a correct result for the far-field. The scalings of the components of the solution $\overline{\mathbf{Y}}$ of (33) as a function of $k$ are summarized in Table III. The rightmost column of the table identifies the current components that can be recovered from $\overline{\mathbf{Y}}$ since do not undergo numerical cancelation, i.e. the components corresponding to the lowest power of $k$ in the real and in the imaginary part of $\overline{\mathbf{Y}}$.

For example, when the excitation is a plane wave, both $R_{s}$ and $I_{n s}$ are necessary to correctly recover the far field. Given that, as it is clear from Table I, only $R_{s}$ is recovered at low frequencies by any method that does not decompose the current, i.e. any method that has the (solenoidal/non-solenoidal undecomposed) physical current as a solution. This is the very low frequency cancelation phenomenon that has been recognized and analyzed in [24]. From Table III, however, it is clear that the new equation recovers all the necessary components to obtain a correct current (recovery of $R_{s}$ ) and a correct far field (recovery of $R_{s}$ and $I_{n s}$ ). Similarly, for the other two classes of sources, it is evident that the rightmost column of Table III always contains the union of the rightmost columns of Tables I and II so that the new equation always recover correctly both physical currents and far-fields. It is worth noticing the role played by the imaginary constant in the definition of the matrix $\bar{M}$ in (26) that ensures that all the necessary components are always recovered by the new equation either in the real or in the imaginary part of $\overline{\mathbf{Y}}$.

\begin{tabular}{lccc}
\hline Source & $\left(R_{s}, I_{s}\right)$ & $\left(R_{n s}, I_{n s}\right)$ & Current Leading term \\
\hline Plane Wave & $(1, k)$ & $\left(k^{2}, k\right)$ & $R_{s}$ \\
Ind. Voltage Gap & $\left(k^{2}, \frac{1}{k}\right)$ & $\left(k^{4}, k\right)$ & $I_{s}$ \\
Cap. Voltage Gap & $\left(k^{4}, k\right)$ & $\left(k^{4}, k\right)$ & $I_{s}, I_{n s}$ \\
\hline
\end{tabular}

TABLE I

SCALings of The Physical CURREnt $\overline{\mathbf{I}}$

\begin{tabular}{lccc}
\hline Source & $\left(k I_{s}, k R_{s}\right)$ & $\left(R_{n s}, I_{n s}\right)$ & Current Leading term \\
\hline Plane Wave & $\left(k^{2}, k\right)$ & $\left(k^{2}, k\right)$ & $R_{s}, I_{n s}$ \\
Ind. Voltage Gap & $\left(1, k^{3}\right)$ & $\left(k^{4}, k\right)$ & $I_{s}$ \\
Cap. Voltage Gap & $\left(k^{2}, k^{5}\right)$ & $\left(k^{4}, k\right)$ & $I_{n s}$ \\
\hline
\end{tabular}

TABLE II

SCALINGS OF THE FAR FIELD

\begin{tabular}{lccc}
\hline Source & $\left(\sqrt{k} R_{s}, \sqrt{k} I_{s}\right)$ & $\left(\frac{I_{n s}}{\sqrt{k}}, \frac{R_{n s}}{\sqrt{k}}\right)$ & Recovered terms \\
\hline Plane Wave & $(\sqrt{k}, k \sqrt{k})$ & $(\sqrt{k}, k \sqrt{k})$ & $R_{s}, I_{n s}, R_{n s}, I_{s}$ \\
Ind. Voltage Gap & $\left(k^{2} \sqrt{k}, \frac{1}{\sqrt{k}}\right)$ & $\left(\sqrt{k}, k^{3} \sqrt{k}\right)$ & $I_{s}, I_{n s}$ \\
Cap. Voltage Gap & $\left(k^{4} \sqrt{k}, k \sqrt{k}\right)$ & $\left(\sqrt{k}, k^{3} \sqrt{k}\right)$ & $I_{s}, I_{n s}$ \\
\hline
\end{tabular}

TABLE III

SCALINGS OF THE SOLUTION $\overline{\mathbf{Y}}$ OF (33)

\section{IMPLEMENTATION RELATED TOPICS}

This section provides further information to stably and rapidly implement the newly proposed equation starting from a standard EFIE code. The implementation presented here allows for a stable use of the new equation (33) from high frequency till arbitrary low frequencies. It is not excluded that the implementation could be simplified if a shorter frequency range of operation is sufficient. For the sake of brevity, however, we will not distinguish between different frequency ranges and we will present the most conservative choices that will allow the implementation to operate in the entire frequency spectrum.

In order to limit the length of the treatment, we will rely on some of the definitions used in [10] for implementing the standard Calderón EFIE. More in detail, in the following we will use the matrices $\overline{\overline{\mathbf{Z}}}^{b}, \overline{\overline{\mathbf{R}}}, \overline{\overline{\mathbf{P}}}$, and $\overline{\overline{\mathbf{G}}}$ whose explicit (and simple) definition can be found in [10]. Moreover we will refer to the matrices $\overline{\overline{\mathbf{Z}}}_{A}^{b}$ and $\overline{\overline{\mathbf{Z}}}_{\phi}^{b}$ that are the vector and scalar potential contributions of $\overline{\mathbf{Z}}^{b}$, with $\overline{\overline{\mathbf{Z}}}^{b}=\overline{\overline{\mathbf{Z}}}_{A}^{b}+\overline{\overline{\mathbf{Z}}}_{\phi}^{b}$. In addition, we define the edge matrix $\overline{\overline{\mathbf{E}}}$ as the $N \times N$ diagonal matrix so that $\overline{\overline{\mathbf{E}}}_{i, i}=l_{i}$ where $l_{i}$ is the length of the edge associated with the RWG $i$. With these definitions we have

$$
k \overline{\overline{\mathbf{Z}}}_{s}=\overline{\overline{\mathbf{P}}}^{T} \overline{\overline{\mathbf{Z}}}_{A}^{b} \overline{\overline{\mathbf{P}}} \quad k \overline{\overline{\mathbf{Z}}}_{s}=\overline{\overline{\mathbf{E}}}^{-1} \overline{\overline{\mathbf{R}}}^{T} \overline{\overline{\mathbf{Z}}}_{A}^{b} \overline{\overline{\mathbf{R}}} \overline{\overline{\mathbf{E}}}^{-1}
$$




$$
\begin{aligned}
& \overline{\overline{\mathbb{Z}}}_{h}=k \overline{\overline{\mathbf{P}}}^{T} \overline{\overline{\mathbf{Z}}}_{\phi}^{b} \overline{\overline{\mathbf{P}}} \quad \overline{\overline{\mathbf{Z}}}_{h}=k \overline{\overline{\mathbf{E}}}^{-1} \overline{\overline{\mathbf{R}}}^{T} \overline{\overline{\mathbf{Z}}}_{\phi}^{b} \overline{\overline{\mathbf{R}}} \overline{\overline{\mathbf{E}}}^{-1} \\
& \overline{\overline{\mathbf{G}}}_{m i x}^{-1}=\left(\overline{\overline{\mathbf{R}}}^{T} \overline{\overline{\mathbf{G}}} \overline{\overline{\mathbf{P}}}\right)^{-1} \\
& \overline{\overline{\mathbf{A}}}_{1}=\left(\overline{\overline{\mathbf{M}}}\left(k \overline{\overline{\mathbf{Z}}}_{s}\right) \overline{\overline{\mathbf{M}}}\right) \overline{\overline{\mathbf{G}}}_{m i x}^{-1}\left(\overline{\overline{\mathbf{M}}}\left(k \overline{\overline{\mathbf{Z}}}_{s}\right) \overline{\overline{\mathbf{M}}}\right) \\
& \overline{\overline{\mathbf{A}}}_{2}=\left(-\overline{\overline{\mathbb{P}}}^{\Lambda}\left(\overline{\overline{\mathbb{Z}}}_{h}\right) \overline{\overline{\mathbb{P}}}^{\Lambda}\right) \overline{\overline{\mathbf{G}}}_{\text {mix }}^{-1}\left(\overline{\overline{\mathbf{M}}}\left(k \overline{\overline{\mathbf{Z}}}_{s}\right) \overline{\overline{\mathbf{M}}}\right) \\
& =\left(-\overline{\overline{\mathbb{P}}}^{\Lambda}\left(\overline{\overline{\mathbb{Z}}}_{h}\right) \overline{\overline{\mathbb{P}}}^{\Lambda}\right) \overline{\overline{\mathbf{G}}}_{m i x}^{-1}\left(\left(\sqrt{k} \overline{\overline{\mathbf{Z}}}_{s}\right) \overline{\overline{\mathbf{M}}}\right) \\
& \overline{\overline{\mathbf{A}}}_{3}=\left(\overline{\overline{\mathrm{M}}}\left(k \overline{\overline{\mathbf{Z}}}_{s}\right) \overline{\overline{\mathbf{M}}}\right) \overline{\overline{\mathbf{G}}}_{m i x}^{-1}\left(-\overline{\overline{\mathbf{P}}}^{\Sigma}\left(\overline{\overline{\mathbf{Z}}}_{h}\right) \overline{\overline{\mathbf{P}}}^{\Sigma}\right) \\
& =\left(\overline{\overline{\mathbf{M}}}\left(\sqrt{k} \overline{\overline{\mathbb{Z}}}_{s}\right)\right) \overline{\overline{\mathbf{G}}}_{m i x}^{-1}\left(-\overline{\overline{\mathbf{P}}}^{\Sigma}\left(\overline{\overline{\mathbf{Z}}}_{h}\right) \overline{\overline{\mathbf{P}}}^{\Sigma}\right) \\
& \overline{\mathbf{V}}_{1}=\left(\overline{\overline{\mathbf{M}}}\left(k \overline{\overline{\mathbb{Z}}}_{s}\right) \overline{\overline{\mathbf{M}}}\right) \overline{\overline{\mathbf{G}}}_{m i x}^{-1} \frac{1}{\sqrt{k}} \overline{\overline{\mathbf{P}}}^{\Lambda H} \overline{\mathbf{V}} \\
& -\left(\overline{\overline{\mathbb{P}}}^{\Lambda}\left(\overline{\overline{\mathbb{Z}}}_{h}\right) \overline{\overline{\mathbb{P}}}^{\Lambda}\right) \overline{\overline{\mathbf{G}}}_{m i x}^{-1} \frac{1}{\sqrt{k}} \overline{\overline{\mathbf{P}}}^{\Lambda H} \overline{\mathbf{V}} \\
& \overline{\mathbf{V}}_{2}=\left(\overline{\overline{\mathrm{M}}}\left(k \overline{\overline{\mathbb{Z}}}_{s}\right) \overline{\overline{\mathrm{M}}}\right) \overline{\overline{\mathbf{G}}}_{m i x}^{-1} \sqrt{k} \overline{\overline{\mathbf{P}}}{ }^{\Sigma} \overline{\mathbf{V}} \\
& =\left(\overline{\overline{\mathrm{M}}}\left(k \overline{\overline{\mathbf{Z}}}_{s}\right)\right) \overline{\overline{\mathbf{G}}}_{m i x}^{-1} \overline{\overline{\mathbf{P}}}^{\Sigma} \overline{\mathbf{V}}
\end{aligned}
$$

After solving the system

$$
\left(\overline{\overline{\mathbf{A}}}_{1}+\overline{\overline{\mathbf{A}}}_{2}+\overline{\overline{\mathbf{A}}}_{3}\right) \overline{\mathbf{Y}}=\overline{\mathbf{V}}_{1}+\overline{\mathbf{V}}_{2}
$$

the solution vector $\overline{\mathbf{I}}$ is recovered as

$$
\begin{aligned}
\overline{\mathbf{I}}_{s} & =\frac{1}{\sqrt{k}}\left(\overline{\overline{\mathbf{P}}}^{\Lambda H} \overline{\mathbf{Y}}\right) \\
\overline{\mathbf{I}}_{n s} & =i \sqrt{k}\left(\overline{\overline{\mathbf{P}}}^{\Sigma} \overline{\mathbf{Y}}\right) \\
\overline{\mathbf{I}} & =\overline{\mathbf{I}}_{s}+\overline{\mathbf{I}}_{n s}
\end{aligned}
$$

The usual techniques used when dealing with very low frequency problems should be used over here. A particular care should be devoted in evaluating (45). As pointed out in [36] when $\overline{\mathbf{V}}$ is generated by the plane wave $e^{i k \hat{k} \cdot \boldsymbol{r}}$, in order to avoid unstabilities, the vector $\overline{\mathbf{V}}$ in (45) should be replaced by the vector $\overline{\mathbf{V}}_{\text {ext }}$ generated by $\left(e^{i k \hat{k} \cdot \boldsymbol{r}}-1\right)$ with the standard techniques to avoid numerical cancelation. A similar caution should be used when calculating the scattered far field. The field should be calculated from $\overline{\mathbf{I}}_{n s}$ and $\overline{\mathbf{I}}_{s}$ separately and in the latter the kernel of the radiation integral should be $\left(e^{i k \hat{k} \cdot \boldsymbol{r}}-1\right)$. These techniques are standard and we will not provide further details that can be found, for example, in [36]. Similar cautions should be taken for the other two typologies of excitation. When the excitation is a capacitive voltage gap, $\overline{\mathbf{V}}_{1}$ should be put to zero to avoid numerical cancelation. Moreover, the projection $\overline{\overline{\mathbf{P}}}^{\Lambda H} \overline{\mathbf{Y}}$ in (48) should always be done accurately. In particular, $\overline{\mathbf{P}}^{\Lambda H} \overline{\mathbf{Y}}$ should be replaced by an exact zero anytime $\left\|\overline{\mathbf{P}}^{\Lambda H} \overline{\mathbf{Y}}\right\| /\|\overline{\mathbf{Y}}\|$ is in the order of the machine precision.

We note that when dealing with open structures, the appropriate definition of $\overline{\overline{\mathbf{P}}}$ (which makes use of half barycentric RWGs) should be used [10]. Likewise, when treating structures

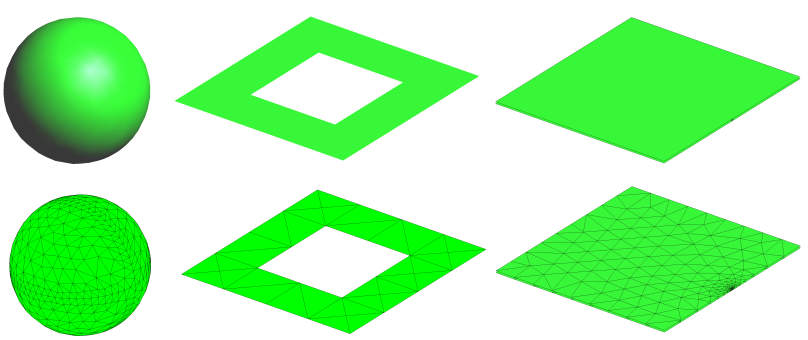

Fig. 2. Geometries used in testing the new equation: sphere, square inductor, and parallel plate capacitor.

with geometric junctions, the same strategies that can be used with standard Calderón techniques [37] can be used here.

We conclude the section by explaining how to efficiently compute the pseudoinverses $\left(\overline{\bar{\Lambda}}^{T} \overline{\bar{\Lambda}}\right)^{+}$and $\left(\overline{\bar{\Sigma}}^{T} \overline{\bar{\Sigma}}\right)^{+}$. The first step is to deflect the matrices in order to transform the pseudoinverse into a standard inverse. In fact it is easy to show that

$$
\begin{aligned}
\left(\overline{\overline{\boldsymbol{\Sigma}}}^{T} \overline{\overline{\mathbf{\Sigma}}}+\frac{1}{N_{c}} \overline{\mathbf{1}}^{\Sigma}\left(\overline{\mathbf{1}}^{\Sigma}\right)^{T}\right)^{+} & =\left(\overline{\overline{\mathbf{\Sigma}}}^{T} \overline{\overline{\mathbf{\Sigma}}}+\frac{1}{N_{c}} \overline{\mathbf{1}}^{\Sigma}\left(\overline{\mathbf{1}}^{\Sigma}\right)^{T}\right)^{-1} \\
& =\left(\overline{\overline{\boldsymbol{\Sigma}}}^{T} \overline{\overline{\mathbf{\Sigma}}}\right)^{+}+\frac{1}{N_{c}} \overline{\mathbf{1}}^{\Sigma}\left(\overline{\mathbf{1}}^{\Sigma}\right)^{T}
\end{aligned}
$$

then, since $\overline{\bar{\Sigma}}\left(\overline{\mathbf{1}}^{\Sigma}\left(\overline{\mathbf{1}}^{\Sigma}\right)^{T}\right) \overline{\bar{\Sigma}}^{T}=\overline{\overline{\mathbf{0}}}$, we have

$$
\overline{\overline{\boldsymbol{\Sigma}}}\left(\overline{\overline{\boldsymbol{\Sigma}}}^{T} \overline{\overline{\boldsymbol{\Sigma}}}\right)^{+} \overline{\overline{\boldsymbol{\Sigma}}}^{T}=\overline{\overline{\boldsymbol{\Sigma}}}\left(\overline{\overline{\boldsymbol{\Sigma}}}^{T} \overline{\overline{\mathbf{\Sigma}}}+\frac{1}{N_{c}} \overline{\mathbf{1}}^{\Sigma}\left(\overline{\mathbf{1}}^{\Sigma}\right)^{T}\right)^{-1} \overline{\overline{\boldsymbol{\Sigma}}}^{T}
$$

and dually

$$
\overline{\overline{\mathbf{\Lambda}}}\left(\overline{\overline{\mathbf{\Lambda}}}^{T} \overline{\overline{\mathbf{\Lambda}}}\right)^{+} \overline{\overline{\mathbf{\Lambda}}}^{T}=\overline{\overline{\mathbf{\Lambda}}}\left(\overline{\overline{\mathbf{\Lambda}}}^{T} \overline{\overline{\mathbf{\Lambda}}}+\frac{1}{N_{v}} \overline{\mathbf{1}}^{\Lambda}\left(\overline{\mathbf{1}}^{\Lambda}\right)^{T}\right)^{-1} \overline{\overline{\mathbf{\Lambda}}}^{T}
$$

note however that the latter is strictly necessary only when $\overline{\bar{\Lambda}}^{T} \overline{\bar{\Lambda}}$ is singular (on closed structures for example). Since the eigenvectors of the matrices $\left(\overline{\bar{\Lambda}}^{T} \overline{\bar{\Lambda}}+\frac{1}{N_{v}} \overline{\mathbf{1}}^{\Lambda}\left(\overline{\mathbf{1}}^{\Lambda}\right)^{T}\right)^{-1}$ and $\left(\overline{\overline{\boldsymbol{\Sigma}}}^{T} \overline{\overline{\boldsymbol{\Sigma}}}+\frac{1}{N_{c}} \overline{\mathbf{1}}^{\Sigma}\left(\overline{\mathbf{1}}^{\Sigma}\right)^{T}\right)^{-1}$ are those of a graph Laplacian, standard multigrids provide optimal preconditioning and both matrices can be inverted in linear time (further details on this can be found in [11]). Note moreover, that if $\Gamma$ is made out of multiple scatterers, the generalization of the formulas above is straightforward. The graph Laplacian should be deflected with a number of vectors equal to the number of disconnected components of $\Gamma$ in the case of $\bar{\Sigma}^{T} \overline{\bar{\Sigma}}$ and to the number of closed disconnected components of $\Gamma$ in the case of $\overline{\bar{\Lambda}}^{T} \overline{\bar{\Lambda}}$. Each of these vectors is unitary and constant on the corresponding connected component while it is zero on all the others.

\section{NUMERICAL RESULTS}

The new equation was tested for plane wave, inductive, and capacitive excitations of a sphere, a planar square ring, and a two plate system, respectively (see Fig. 2). The first test involves a sphere of unit radius that is excited by a plane wave. Figs. 3 and 4 show the condition number of the matrix on the 


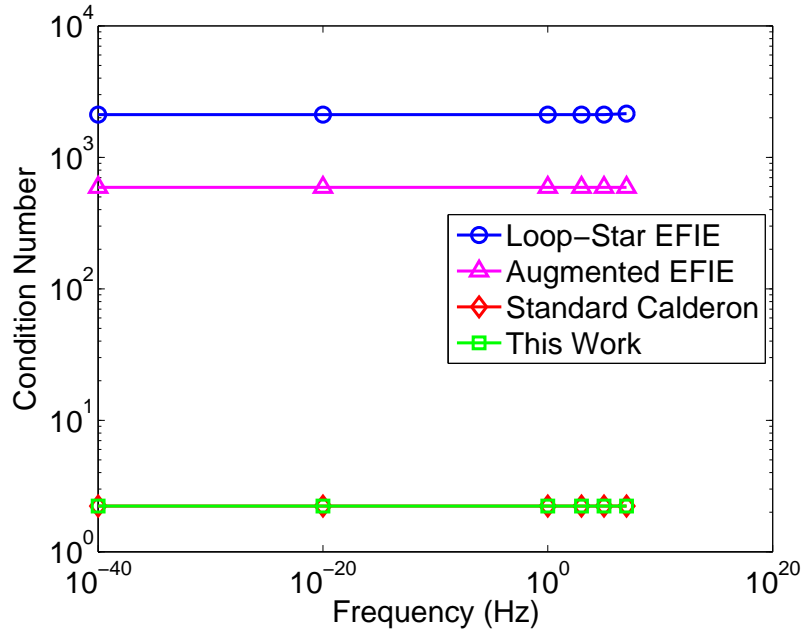

Fig. 3. Sphere: condition number of (33) as a function of the excitation frequency.

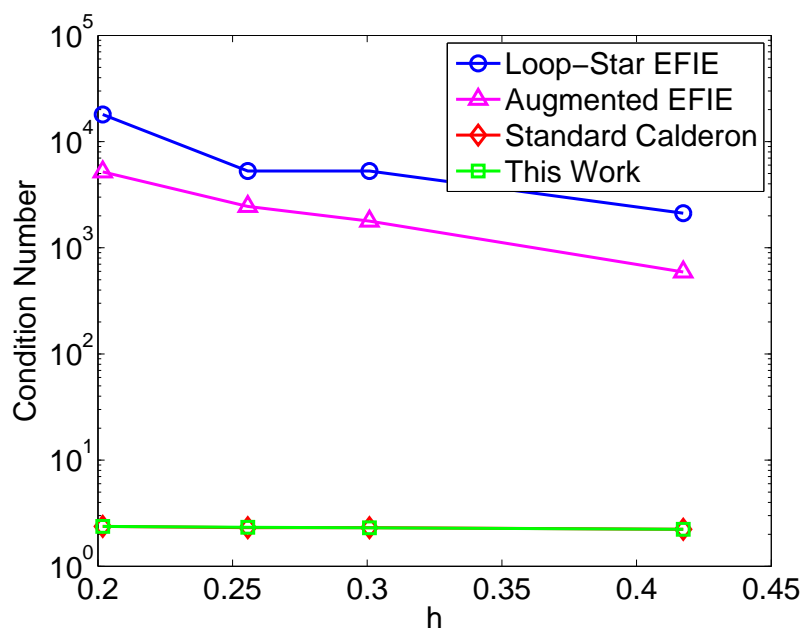

Fig. 4. Sphere: condition number of (33) as a function of the average mesh size $h$.

left hand side of (33) as a function of the excitation frequency and the discretization density, respectively. The proposed equation clearly is immune from low frequency and dense discretization breakdown. The behavior of the equation is also compared with that of Loop-Star-decomposed and Augmented EFIEs ([12], equation (9)). In Fig. 3, for the Augmented EFIE, the three smallest frequencies $\left(f=1 \mathrm{~Hz}, f=10^{-20} \mathrm{~Hz}\right.$, and $f=10^{-40} \mathrm{~Hz}$ ) produce convergent, but incorrect solutions. This is because, as explained in [26], at very low frequencies the Augmented EFIE should be coupled with a perturbation method. The star component of the current for a frequency of $10^{-40} \mathrm{~Hz}$ computed using (33) is shown in Fig. 5; the loop component is shown in Fig. 6. Each time, the current obtained by solving (33) is compared to that obtained by solving a standard Loop-Star EFIE, a formulation that is known to provide the correct current in both the solenoidal and the non-solenoidal components, although it is difficult to solve as it suffers from dense discretization breakdown as it is clear from Fig. 4. From these two figures it is clear that the new equation is immune from the very low frequency current

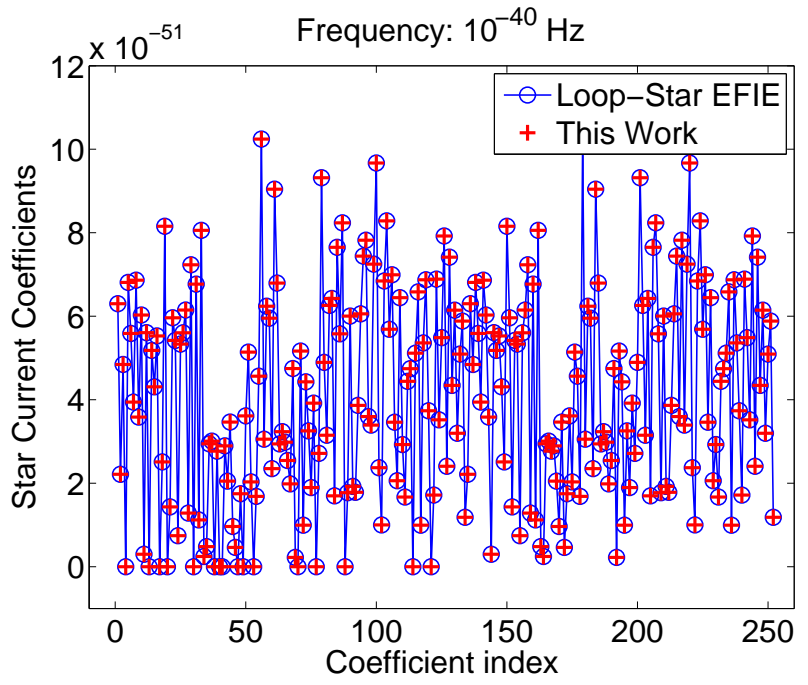

Fig. 5. Sphere, frequency: $10^{-40} \mathrm{~Hz}$. Comparison of the Star component of the induced current calculated with (33) and with a Loop-Star EFIE.

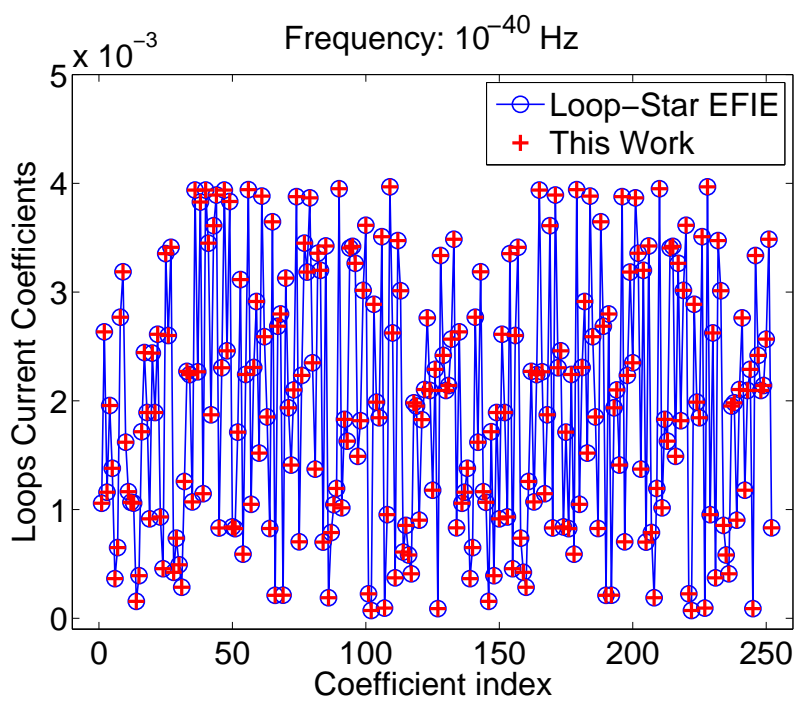

Fig. 6. Sphere, frequency: $10^{-40} \mathrm{~Hz}$. Comparison of the Loop component of the induced current calculated with (33) and with a Loop-Star EFIE.

cancelation. This is also confirmed by Fig. 7 and Fig. 8 which show the far field calculated using (33) for frequencies of $1 \mathrm{~Hz}$ and $10^{-40} \mathrm{~Hz}$, respectively. The solution of (33) is in good agreement with the solution of the standard Calderón EFIE as well as with the Mie series result. It is clear that although a standard Calderón equation can provide a stable solution till low frequencies $(1 \mathrm{~Hz})$, the new equation (33) is immune from the very low frequency current cancelation and provides stable solutions even when the frequency is arbitrarily low.

To study the behavior of the new equation (33) when applied to inductive structures with voltage gap excitations, consider the square ring with side length $1 \mathrm{~m}$ and width $0.25 \mathrm{~m}$ shown in Fig. 2); the voltage gap is located in the center of one of the ring's sides. Fig. 9 shows the absolute value of the input inductance as a function of frequency. The values obtained by solving (33) are in very good agreement with those obtained using the standard Loop-Star EFIE. The computed 


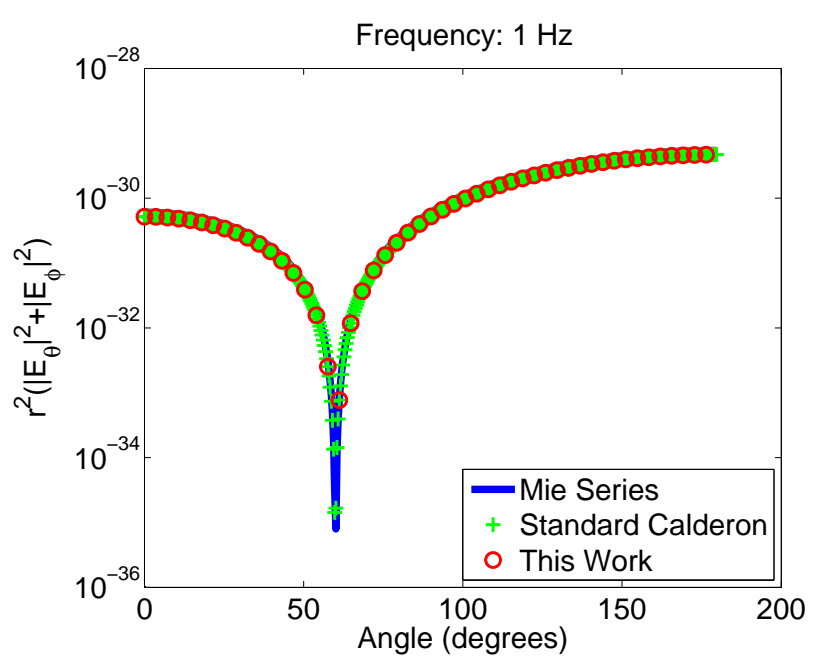

Fig. 7. Sphere: far field calculated when the frequency equals $1 \mathrm{~Hz}$

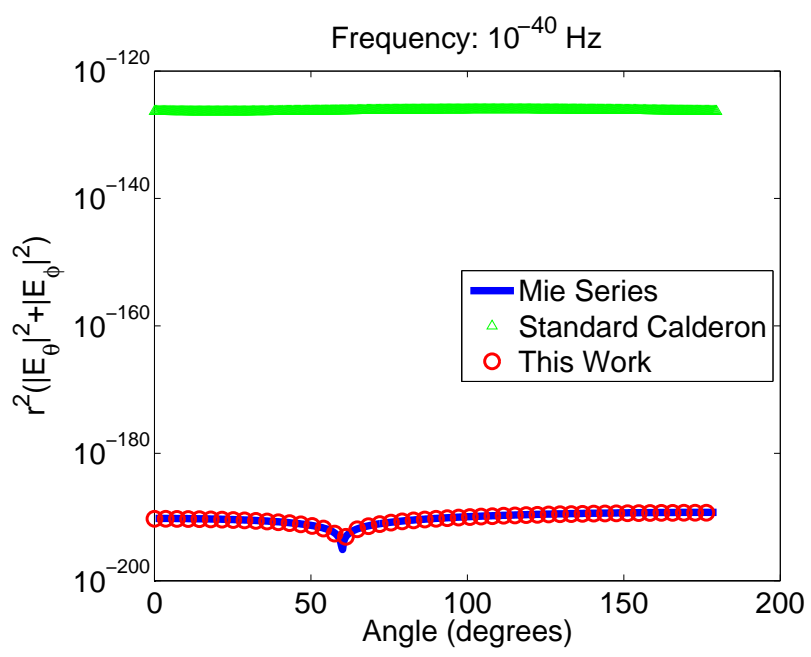

Fig. 8. Sphere: far field calculated when the frequency equals $10^{-40} \mathrm{~Hz}$

value of the static inductance is near $1.197 \mu \mathrm{H}$, the approximate inductance value predicted using classical expressions. The ring's far fields for a frequency of $10^{-40} \mathrm{~Hz}$ computed using (33) and the Loop-Star EFIE, are shown in Fig. 10 and match very well. Clearly, when the new equation is applied to inductive structures with voltage gap excitations, the far fields do not suffer from very low frequency cancelations. Fig. 11 compares the singular values of the system matrix produced by the standard Calderón approach and (33) for a frequency of $10^{-40} \mathrm{~Hz}$. A static null-space of dimension one is expected for the Calderón EFIO, since the open structure has one hole. An almost zero singular value is evident in Fig. 11. In contrast, and as predicted by our theory, the new equation however does not have a static null-space.

To study the behavior of the new equation (33) when applied to capacitive structures with voltage gap excitations, consider the parallel plate capacitor composed of plates with side length $1 \mathrm{~m}$ that are separated by $1 \mathrm{~cm}$ shown in Fig. 2); a voltage gap is located in a narrow strip that connects the two plates. Fig. 12 shows the absolute value of the input capacitance as

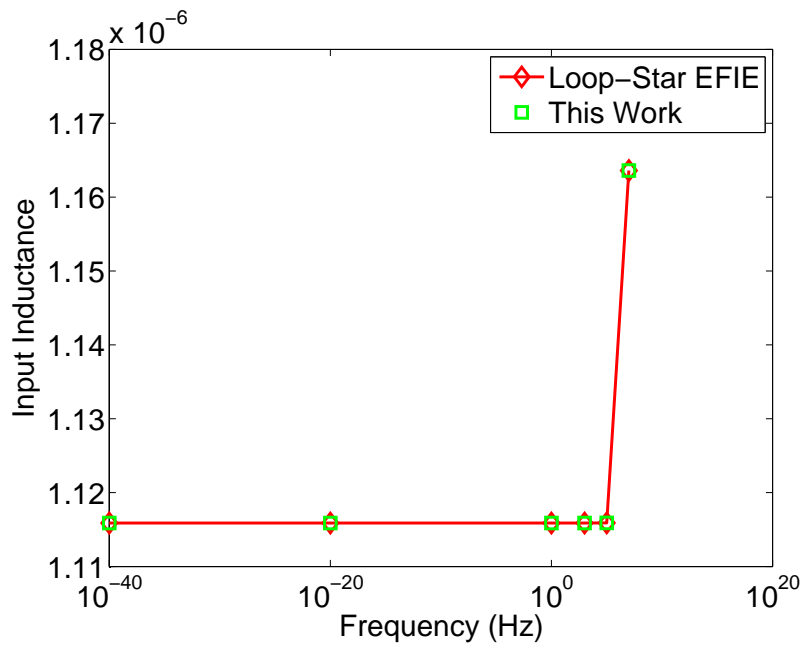

Fig. 9. Input inductance of the square inductor as a function of the frequency.

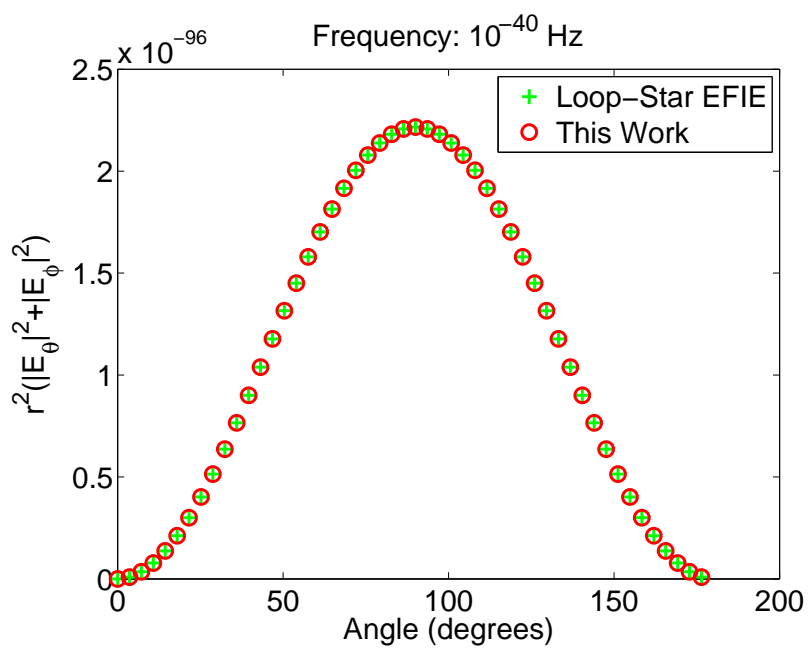

Fig. 10. RCS of the square inductor with voltage gap excitation

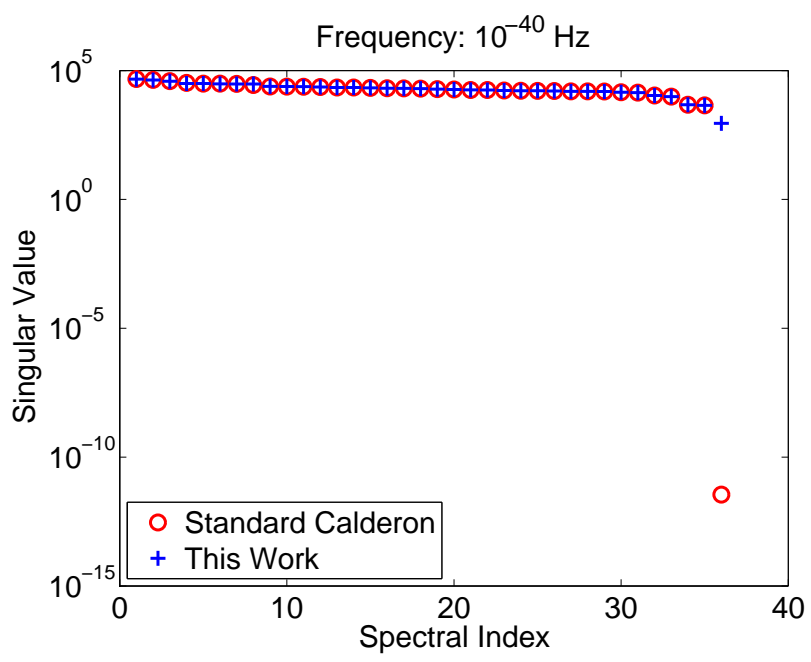

Fig. 11. Square inductor: singular values of the standard Calderón EFIO and of operator in (33). 


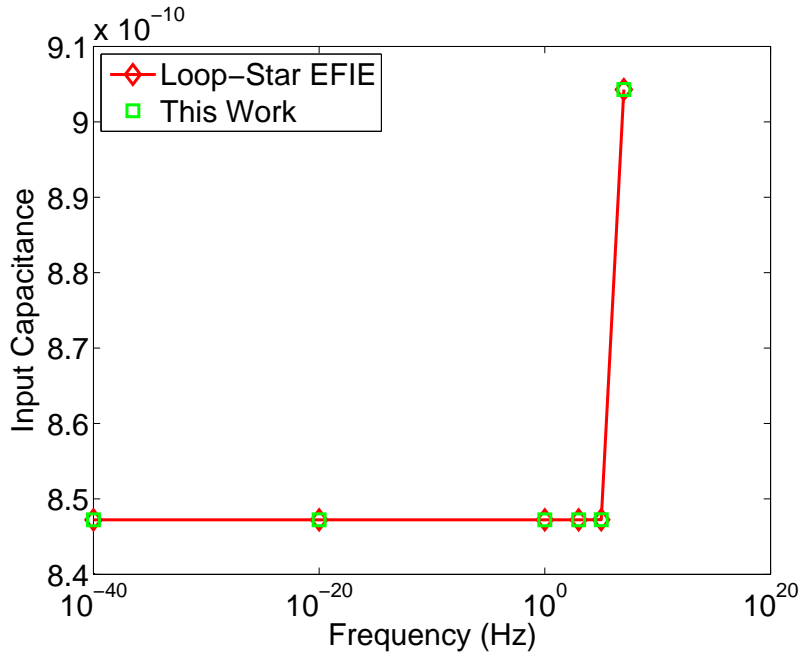

Fig. 12. Input capacitance of the parallel plate capacitor as a function of the frequency.

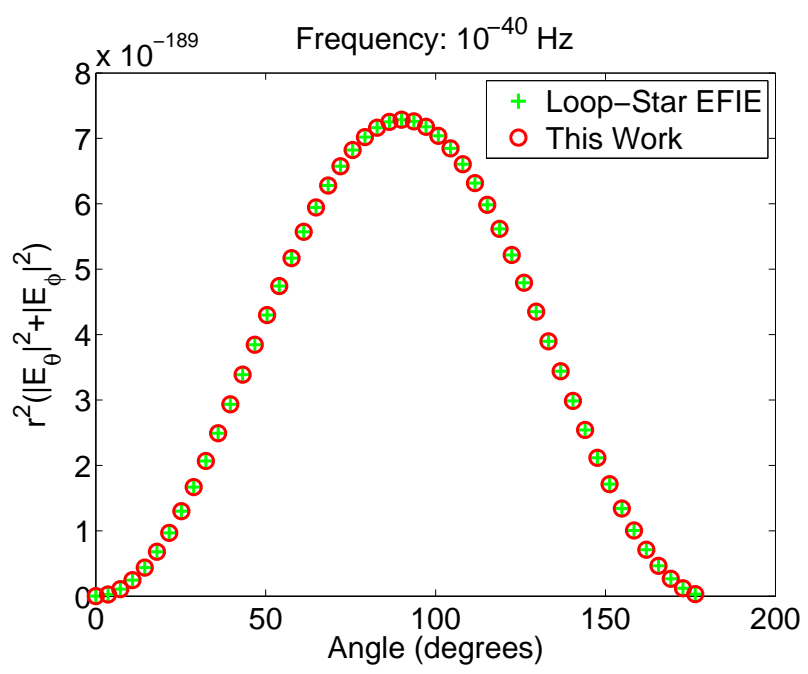

Fig. 13. RCS of the parallel plate capacitor with voltage gap excitation

a function of frequency (the reader should not be puzzled by the abrupt change of the capacitance value in the plot which is solely due to the choice of the axis' scale). The capacitance values obtained by solving (33) are in good agreement with those obtained with a standard Loop-Star EFIE. Moreover, the computed static capacitance is near $0.884 \mathrm{nF}$, the approximate capacitance value predicted by classical expressions.

The capacitor's far fields for a frequency of $10^{-40} \mathrm{~Hz}$ computed using (33) and the Loop-Star EFIE, are shown in Fig. 13 and match very well. Clearly, when the new equation is applied to capacitive structures with voltage gap excitations, the far fields do not suffer from very low frequency cancelations.

\section{Conclusions}

This paper presented an electric field integral equation that is immune from both low-frequency and dense discretization breakdown, does not require a search for global topological loops, and does not suffer from numerical cancelations in the solution when the frequency is very low. The computational cost of all calculations in the new formulation not required by the solvers it builds on scale linearly in the number of unknowns; hence the new formulation can be applied in tandem with fast methods without degradation in computational complexity. Numerical results demonstrated the beneficial properties of the new technique. It should be noted that standard and Calderon EFIOs are not uniquely invertible when defined on closed objects when the frequency corresponds to an internal resonance. This problem is traditionally solved by using combined field operators. It appears that a similar approach can be applied to the new EFIO presented in this work and this is the subject of current research.

\section{REFERENCES}

[1] R. Coifman, V. Rokhlin, and S. Wandzura, "The fast multipole method for the wave equation: a pedestrian prescription," IEEE Antennas and Propagation Magazine, pp. 7-12, 1993.

[2] C.-C. Lu and W. Chew, "A multilevel algorithm for solving a boundary integral equation of wave scattering," Microwave Opt. Technol. Lett., vol. 7, no. 10, pp. 466-470, 1994.

[3] E. Michielssen and A. Boag, "A multilevel matrix decomposition algorithm for analyzing scattering from large structures," IEEE Transactions on Antennas and Propagation, vol. 44, no. 8, pp. 1086-1093, Aug. 1996.

[4] F. Andriulli, A. Tabacco, and G. Vecchi, "Solving the EFIE at low frequencies with a conditioning that grows only logarithmically with the number of unknowns," IEEE Transactions on Antennas and Propagation, vol. 58, no. 5, pp. $1614-1624$, may 2010.

[5] D. R. Wilton and A. W. Glisson, "On improving the stability of the electric field integral equation at low frequencies," USNC/URSI Spring Meeting Digest, p. 24, 1981.

[6] G. Vecchi, "Loop-star decomposition of basis functions in the discretization of the EFIE," IEEE Transactions on Antennas and Propagation, vol. 47, no. 2, pp. 339-346, Feb. 1999.

[7] J. S. Zhao and W. C. Chew, "Integral equation solution of Maxwell's equations from zero frequency to microwave frequencies," IEEE Transactions on Antennas and Propagation, vol. 48, no. 10, pp. 1635-1645, Oct. 2000.

[8] J.-F. Lee, R. Lee, and R. J. Burkholder, "Loop star basis functions and a robust preconditioner for EFIE scattering problems," IEEE Transactions on Antennas and Propagation, vol. 51, no. 8, pp. 1855-1863, Aug. 2003.

[9] T. F. Eibert, "Iterative-solver convergence for loop-star and loop-tree decompositions in method-of-moments solutions of the electric field integral equation," IEEE Antennas and Propagation Magazine, vol. 46, no. 3, pp. $80-85$, Jun. 2004.

[10] F. P. Andriulli, K. Cools, H. Bagci, F. Olyslager, A. Buffa, S. Christiansen, and E. Michielssen, "A multiplicative calderon preconditioner for the electric field integral equation," IEEE Transactions on Antennas and Propagation, vol. 56, no. 8, pp. 2398-2412, 2008.

[11] F. Andriulli, "Loop-star and loop-tree decompositions: analysis and efficient algorithms," IEEE Transactions on Antennas and Propagation, vol. 60 , no. 5, pp. 2347 -2356, May 2012.

[12] Z. G. Qian and W. C. Chew, "An augmented electric field integral equation for high-speed interconnect analysis," Microwave Opt. Technol. Lett., vol. 50, no. 10, pp. 2658-2662, 2008.

[13] J. Zhu, S. Omar, W. Chai, and D. Jiao, "A rigorous solution to the lowfrequency breakdown in the electric field integral equation," in IEEE International Symposium on Antennas and Propagation 2011, july 2011, pp. $3214-3217$.

[14] F. Vipiana, G. Vecchi, and P. Pirinoli, "A multiresolution system of Rao-Wilton-Glisson functions," IEEE Transactions on Antennas and Propagation, vol. 55, no. 3, pp. 924-930, 2007.

[15] F. P. Andriulli, F. Vipiana, and G. Vecchi, "Hierarchical bases for nonhierarchic 3D triangular meshes," IEEE Transactions on Antennas and Propagation, vol. 56, no. 8, pp. 2288-2297, 2008.

[16] R.-S. Chen, J. Ding, D. Ding, Z. Fan, and D. Wang, "A multiresolution curvilinear Rao-Wilton-Glisson basis function for fast analysis of electromagnetic scattering," IEEE Transactions on Antennas and Propagation, vol. 57, no. 10, pp. 3179 -3188, Oct. 2009.

[17] S. H. Christiansen and J.-C. Nedéléc, "A preconditioner for the electric field integral equation based on Calderon formulas," SIAM J. Num. Anal., vol. 40, no. 3, pp. 1100-1135, 2002. 
[18] H. Contopanagos, B. Dembart, M. Epton, J. Ottusch, V. Rokhlin, J. Visher, and S. M. Wandzura, "Well-conditioned boundary integral equations for three-dimensional electromagnetic scattering," IEEE Transactions on Antennas and Propagation, vol. 50, no. 12, pp. 18241930, Dec. 2002.

[19] R. J. Adams, "Physical and analytical properties of a stabilized electric field integral equation," IEEE Transactions on Antennas and Propagation, vol. 52, no. 2, pp. 362-372, Feb. 2004.

[20] M. Stephanson and J.-F. Lee, "Preconditioned electric field integral equation using Calderon identities and dual loop/star basis functions," IEEE Transactions on Antennas and Propagation, vol. 57, no. 4, pp. 1274-1279, April 2009.

[21] S. Yan, J.-M. Jin, and Z. Nie, "EFIE analysis of low-frequency problems with loop-star decomposition and Calderon multiplicative preconditioner," IEEE Transactions on Antennas and Propagation, vol. 58, no. 3, pp. $857-867$, Mar. 2010.

[22] C. L. Epstein and L. Greengard, "Debye sources and the numerical solution of the time harmonic Maxwell equations," Communications on Pure and Applied Mathematics, vol. 63, no. 4, pp. 413-463, 2010.

[23] K. Cools, F. Andriulli, F. Olyslager, and E. Michielssen, "Nullspaces of MFIE and Calderon preconditioned EFIE operators applied to toroidal surfaces," IEEE Transactions on Antennas and Propagation, vol. 57, no. 10 , pp. $3205-3215$, Oct. 2009.

[24] Y. Zhang, T. J. Cui, W. C. Chew, and J.-S. Zhao, "Magnetic field integral equation at very low frequencies," IEEE Transactions on Antennas and Propagation, vol. 51, no. 8, pp. 1864 - 1871, Aug. 2003.

[25] W. C. Chew, M. S. Tong, and B. Hu, "Integral equation methods for electromagnetic and elastic waves," Synthesis Lectures on Computational Electromagnetics, vol. 3, no. 1, pp. 1-241, 2008.

[26] Z.-G. Qian and W. C. Chew, "Enhanced A-EFIE with perturbation method," IEEE Transactions on Antennas and Propagation, vol. 58, no. 10 , pp. $3256-3264$, Oct. 2010 .

[27] I. Bogaert, K. Cools, F. Andriulli, J. Peeters, and D. De Zutter, "Low frequency stability of the mixed discretization of the MFIE," in Proceedings of the 5th European Conference on Antennas and Propagation (EUCAP), Apr. 2011, pp. $2463-2465$.

[28] I. Bogaert, K. Cools, F. Andriulli, and D. De Zutter, "Low frequency scaling of the mixed MFIE for scatterers with a non-simply connected surface," in International Conference on Electromagnetics in Advanced Applications (ICEAA), 2011, Sep. 2011, pp. 951 -954.

[29] S. Sun, W. C. Chew, Y. G. Liu, and Z. Ma, "Perturbation method for lowfrequency Calderon multiplicative preconditioned EFIE," in Proceedings of ACES conference, 2012.

[30] S. M. Rao, D. R. Wilton, and A. W. Glisson, "Elettromagnetic scattering by surfaces of arbitrary shape," IEEE Transactions on Antennas and Propagation, vol. 30, no. 3, pp. 409-418, May 1982.

[31] A. Buffa and S. H. Christiansen, "A dual finite element complex on the barycentric refinement," Math. Comp., vol. 76, no. 260, pp. 1743-1769, 2007.

[32] K. Cools, F. Andriulli, F. Olyslager, and E. Michielssen, "Time domain Calderon identities and their application to the integral equation analysis of scattering by PEC objects part I: Preconditioning," IEEE Transactions on Antennas and Propagation, vol. 57, no. 8, pp. 2352 -2364, Aug. 2009.

[33] A. Buffa and R. Hiptmair, "Galerkin boundary element methods for electromagnetic scattering," in Topics in computational wave propagation, ser. Lect. Notes Comput. Sci. Eng. Berlin: Springer, 2003, vol. 31, pp. 83-124.

[34] J.-C. Nedéléc, Acoustic and Electromagnetic Equations. Springer, 2000.

[35] F. Andriulli, K. Cools, F. Olyslager, and E. Michielssen, "Time domain Calderon identities and their application to the integral equation analysis of scattering by PEC objects part II: stability," IEEE Transactions on Antennas and Propagation, vol. 57, no. 8, pp. 2365 -2375, Aug. 2009.

[36] J.-S. Zhao, W. C. Chew, T. Cui, and Y. Zhang, "Cancellations of surface loop basis functions," in Antennas and Propagation Society International Symposium, 2002. IEEE, vol. 1, 2002, pp. 58 - 61 vol.1.

[37] X. Claeys and R. Hiptmair, "Electromagnetic scattering at composite objects : a novel smulti-trace boundary integral formulation," ESAIM: Mathematical Modelling and Numerical Analysis, vol. 46, no. 06, pp. 1421-1445, 2012. 